\title{
Guide RNA acrobatics: positioning consecutive uridines for pseudouridylation by H/ACA pseudouridylation loops with dual guide capacity
}

\author{
Beáta E. Jády, ${ }^{1}$ Amandine Ketele, ${ }^{1}$ Dylan Moulis, ${ }^{1}$ and Tamás Kiss ${ }^{1,2}$ \\ ${ }^{1}$ Molecular, Cellular, and Developmental Biology Department (MCD) UMR 5077, Centre de Biologie Intégrative (CBI), University \\ of Toulouse, Centre National de la Recherche Scientifique, 31062 Toulouse, France; ${ }^{2}$ Biological Research Centre, 6726 Szeged, \\ Hungary
}

Site-specific pseudouridylation of human ribosomal and spliceosomal RNAs is directed by H/ACA guide RNAs composed of two hairpins carrying internal pseudouridylation guide loops. The distal "antisense" sequences of the pseudouridylation loop base-pair with the target RNA to position two unpaired target nucleotides $5^{\prime}-\mathrm{UN}-3^{\prime}$, including the $5^{\prime}$ substrate $U$, under the base of the distal stem topping the guide loop. Therefore, each pseudouridylation loop is expected to direct synthesis of a single pseudouridine $(\Psi)$ in the target sequence. However, in this study, genetic depletion and restoration and RNA mutational analyses demonstrate that at least four human H/ACA RNAs (SNORA53, SNORA57, SCARNA8, and SCARNA1) carry pseudouridylation loops supporting efficient and specific synthesis of two consecutive pseudouridines ( $\Psi \Psi$ or $\Psi \mathrm{N} \Psi)$ in the $28 \mathrm{~S}(\Psi 3747 / \Psi 3749), 18 \mathrm{~S}(\Psi 1045 / \Psi 1046)$, and U2 $(\Psi 43 / \Psi 44$ and $\Psi 89 / \Psi 91)$ RNAs, respectively. In order to position two substrate Us for pseudouridylation, the dual guide loops form alternative base-pairing interactions with their target RNAs. This remarkable structural flexibility of dual pseudouridylation loops provides an unexpected versatility for RNA-directed pseudouridylation without compromising its efficiency and accuracy. Besides supporting synthesis of at least $6 \%$ of human ribosomal and spliceosomal $\Psi \mathrm{s}$, evidence indicates that dual pseudouridylation loops also participate in pseudouridylation of yeast and archaeal rRNAs.

[Keywords: pseudouridine; guide RNA acrobatics; box H/ACA RNAs; RNA-guided RNA modification; pseudouridylation; RNA-RNA interaction]

Supplemental material is available for this article.

Received September 28, 2021; revised version accepted November 16, 2021.

Mature cellular RNAs carry $>160$ different types of posttranscriptionally modified nucleotides (Boccaletto et al. 2018). The most prevalent modified nucleotide is pseudouridine $(\Psi)$, the 5-ribosyl isomer of $U$, which is present in transfer RNAs (tRNAs), ribosomal RNAs (rRNAs), small nuclear RNAs (snRNAs), and also in polyadenylated mRNAs (Reddy and Busch 1988; Bakin and Ofengand 1998; Carlile et al. 2014; Schwartz et al. 2014; Spenkuch et al. 2014; Li et al. 2015; Taoka et al. 2018). U and $\Psi$ share identical Watson-Crick faces, but the endocyclic N1 group of $\Psi$ can function as an extra proton donor supporting water-coordinated hydrogen bonding that increases the thermodynamic stability of base stacking and the rigidity of the phosphodiester backbone (Arnez and Steitz 1994; Davis 1995; Newby and Greenbaum 2002a,b). Cor-

Corresponding author: tamas.kiss@univ-tlse3.fr

Article published online ahead of print. Article and publication date are online at http://www.genesdev.org/cgi/doi/10.1101/gad.349072.121. Freely available online through the Genes \& Development Open Access option. rect pseudouridylation of spliceosomal snRNAs, rRNAs, and tRNAs is important for efficient and accurate premRNA splicing and protein synthesis (Ge and Yu 2013; Spenkuch et al. 2014; De Zoysa and Yu 2017; Sloan et al. 2017; Bohnsack and Sloan 2018; Adachi et al. 2019). The functional significance of mRNA pseudouridylation is still unclear, but artificially introduced $\Psi$ s can alter stop codon reading, increase mRNA stability and translation efficiency, and suppress RNA immunogenicity (Karikó et al. 2008; Karijolich and Yu 2011; Fernández et al. 2013; Hoernes et al. 2016).

RNA pseudouridylation is achieved by stand-alone and guide RNA-associated $\Psi$ synthases. The stand-alone $\Psi$ synthases recognize local RNA structures and frequently show "region and/or multisite specificity" (Spenkuch et al. 2014). Site-specific synthesis of eukaryotic

(C) 2022 Jády et al. This article, published in Genes \& Development, is available under a Creative Commons License (Attribution-NonCommercial 4.0 International), as described at http://creativecommons.org/licenses/by-nc/4.0/. 
spliceosomal and eukaryotic and archaeal ribosomal $\Psi$ s located in diverse sequence and structural environments is mediated by $\mathrm{H} / \mathrm{ACA}$ guide RNA-associated $\Psi$ synthases (Ganot et al. 1997a; Ni et al. 1997; Darzacq et al. 2002). Through base-pairing with the target RNA, the guide RNAs provide high sequence specificity for the associated $\Psi$ synthase, making the pseudouridylation reaction independent from local two-dimensional RNA structures. While archaeal H/ACA RNAs contain one to three hairpin structures, eukaryotic H/ACA RNAs are composed of two hairpins that are connected and tailed with short singlestranded sequences carrying the conserved $\mathrm{H}$ (AgAnnA) and ACA (less frequently, AUA) boxes, respectively (Fig. 1A; Balakin et al. 1996; Kiss et al. 1996; Ganot et al. 1997b). The molecular principles of RNA-guided pseudouridylation were established more than two decades

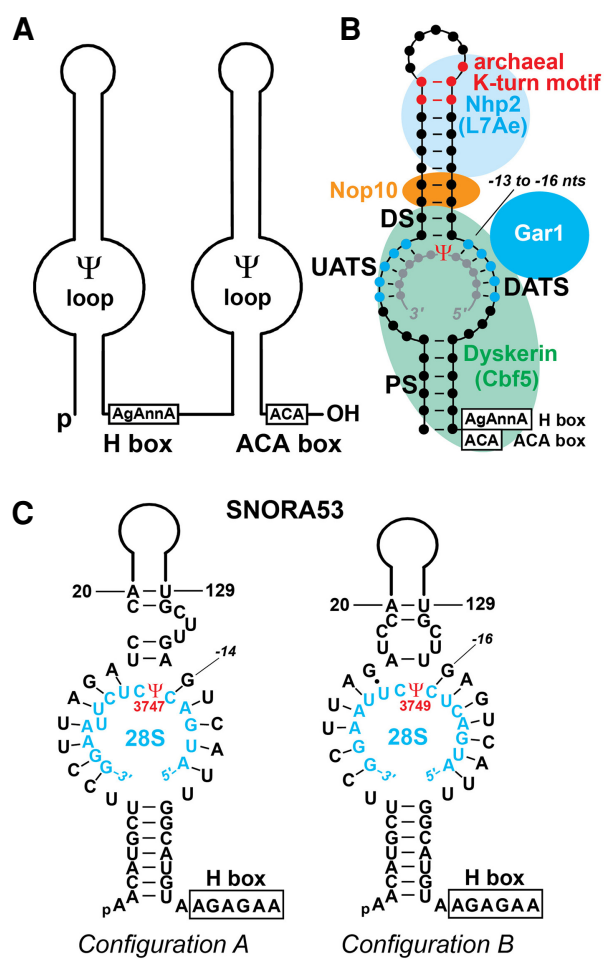

Figure 1. Selection of substrate uridines for pseudouridylation by box H/ACA guide RNAs. (A) Schematic structure of eukaryotic bipartite box H/ACA pseudouridylation guide RNAs. The consensus sequences of the conserved $\mathrm{H}$ and ACA box motifs and the internal pseudouridylation guide loops ( $\Psi$-loop) are shown. $(B)$ Schematic consensus structure of the $5^{\prime}$ and $3^{\prime}$ hairpins of $\mathrm{H} /$ ACA pseudouridylation guide RNPs. The antisense sequences (blue dots) and the selected target sequences (gray dots) with the target $U(\Psi)$ positioned for pseudouridylation are shown. The proximal and distal stems (PS and DS) together with the upstream and downstream antisense target stems (UATS and DATS) are indicated. The four H/ACA core proteins (dyskerin, Nop10, Nhp2, and Gar1) are shown. Archaeal ACA RNAs contain K-turns or K-loops. (C) Potential base-pairing interactions of the proposed 5'-terminal pseudouridylation guide loop of human SNORA53 with 28S rRNA positioning U3747 or U3749 for pseudouridylation. ago (for reviews, see Kiss et al. 2010; Watkins and Bohnsack 2012; Lui and Lowe 2013; Yu and Meier 2014; Czekay and Kothe 2021). The H/ACA hairpins contain internal pseudouridylation guide loops carrying bipartite target recognition (antisense) elements preceding and following the distal stem (DS) that closes the pseudouridylation loop (Ganot et al. 1997a). Base-pairing of the bipartite antisense sequences with the substrate RNA forms an RNA three-way helical junction positioning two unpaired substrate nucleotides $\left(5^{\prime}-\mathrm{UN}-3^{\prime}\right)$ into the "pseudouridylation pocket" at the base of the DS (Fig. 1B). Invariantly, the unpaired $5^{\prime} \mathrm{U}$ that is separated by $13-16 \mathrm{nt}$ from the $\mathrm{H}$ or ACA box of the guide RNA is converted into $\Psi$.

Each H/ACA hairpin associates with four evolutionarily conserved proteins: Nop10, Gar1, Nhp2 (L7Ae in archaea), and the $\Psi$ synthase dyskerin (also called NAP57 in mice and Cbf5 in yeast and archaea) (Fig. 1B). First, the structure of archaeal single-hairpin ACA RNP has been solved (Li and Ye 2006; Liang et al. 2007, 2009; Duan et al. 2009). The ACA RNA hairpin is anchored to the core trimer of Cbf5, Nop10, and L7Ae through bindings of Cbf5 to the ACA box and the proximal stem (PS) and L7Ae to the kink (K)-turn or K-loop in the apical stem-loop. These interactions position the selected target $\mathrm{U}$ at the catalytic center of Cbf5. Garl associates with the catalytic domain of Cbf5 and controls its activity through promoting accurate substrate placing and releasing (Duan et al. 2009; Wang et al. 2015). Eukaryotic double-hairpin H/ACA RNPs have similar structural organization (Egan and Collins 2010; Li et al. 2011). In contrast to archaeal L7Ae, eukaryotic Nhp2 binds to the distal stem-loop independently from a K-turn motif. While Nhp2 alone has little H/ACA RNA binding affinity, upon incorporation into the dyskerin-Nop10-Nhp2 core complex, it binds to the distal stem-loop with high affinity and specificity (Wang and Meier 2004; Baker et al. 2005; Li et al. 2011). Eukaryotic H/ACA RNPs directing rRNA pseudouridylation accumulate in the nucleolus and are called small nucleolar RNPs (snoRNPs). Guide RNPs mediating snRNA pseudouridylation concentrate in the nucleoplasmic Cajal body (CB) and are termed small CB-specific RNPs (scaRNPs) (Darzacq et al. 2002).

Due to their stringent target selection strategy, the $\mathrm{H} /$ ACA pseudouridylation guide loops are believed to position only one substrate $U$ for pseudouridylation in their target sequences (Fig. 1B). However, arguing against this notion, the $3^{\prime}$-terminal pseudouridylation loops of Xenopus pugU2-43, yeast snR82, and archaeal sR-h45 $\mathrm{H} / \mathrm{ACA}$ RNAs have been implicated in synthesis of two consecutive spliceosomal and ribosomal $\Psi$ s $(\Psi \Psi$ and $\Psi N \Psi$ ) through unclear mechanisms (Schattner et al. 2004; Deryusheva and Gall 2013, 2017; Majumder et al. 2020). Here, functional characterization of human $\mathrm{H} / \mathrm{ACA}$ RNAs reveals that at least four human H/ACA RNAs possess pseudouridylation loops with dual guide capacity. We demonstrate that the $5^{\prime}$-terminal pseudouridylation loop of SNORA53 and the $3^{\prime}$-terminal pseudouridylation loops of SNORA57, SCARNA8, and SCARNA1 direct formation of $\Psi 3747 / \Psi 3949$ in the $28 \mathrm{~S}$ rRNA, $\Psi 1045 / \Psi 1046$ in the $18 S$ rRNA, and $\Psi 43 / \Psi 44$ 
and $\Psi 89 / \Psi 91$ in the U2 spliceosomal snRNA, respectively. A detailed mutational analysis of SNORA53, SNORA57, and SCARNA1 confirmed that dynamic alternative guide RNA and substrate RNA base-pairing interactions, termed guide RNA acrobatics, support the sequence specificity and efficacy of the dual pseudouridylation guide loops of human H/ACA RNAs. Since the previously reported dual pseudouridylation guide loops of Xenopus pugU2-43, yeast snR82, and archaeal sR-h45 H/ACA RNAs can also form alternative guidetarget interactions that can correctly position their target Us for pseudouridylation, we propose that H/ACA guide RNA acrobatics is an evolutionarily conserved strategy of pseudouridylation guide RNAs for accurate and efficient synthesis of two proximate $\Psi$ s.

\section{Results}

Human SNORA53 is required for synthesis of both $\Psi 3747$ and $\Psi 3749$ in the $28 S$ rRNA

Human SNORA53, originally named ACA53 (Kiss et al. 2004), is a box H/ACA "orphan" snoRNA, as it has not been assigned for RNA modification or for any other cellular function. Closer inspection of SNORA53 revealed that its $5^{\prime}$-terminal hairpin can be folded into two alternative structures accommodating slightly different putative pseudouridylation loops that, in principle, could direct synthesis of $\Psi 3747$ and $\Psi 3749$ in the 28S rRNA (Fig. 1C). Thus far, no H/ACA snoRNAs have been proposed to govern $\Psi 3747$ and $\Psi 3749$ synthesis (Jorjani et al. 2016). However, in the newly suggested SNORA53-28S rRNA interactions, the alleged pseudouridylation loops, instead of being closed by perfect distal stems, are topped by unusually unstable structures encompassing several bulged and unpaired nucleotides. Moreover, in configuration A assigned for synthesis of $\Psi 3747$, the upstream antisense target stem (UATS) is interrupted with the bulged U3742 residue in the $28 \mathrm{~S}$ rRNA sequence. To test SNORA53 participation in $\Psi 3747$ and/or $\Psi 3749$ synthesis, expression of SNORA53 was knocked out in human HAP1 cells. Mammalian box H/ACA guide RNAs are synthesized within introns of protein-coding genes and are processed from the removed and debranched host introns. The human SNORA53 gene is located within the fifth intron of the SLC25A3 gene in sense orientation, indicating that SNORA53 is a canonical intronic snoRNA (Fig. 2A). A fragment of the fifth intron of SLC25A3 encompassing the SNORA53 gene was excised by CRISPR/Cas9 genome editing. The correctness of SNORA53 deletion was verified by PCR amplification and gel electrophoretic analysis of the truncated SLC25A3 gene fragment (Fig. 2B). RTPCR detected comparable levels of correctly spliced mature SLC25A mRNA in the SNORA53 knockout (KO) and control HAP1 cells, demonstrating that the removal of the SNORA53 gene had no detectable impact on the expression of the SLC25A3 host gene (Fig. 2C). Finally, RNase A/T1 mappings performed with an internally labeled sequence-specific RNA probe failed to detect SNORA53 expression in SNORA53-KO cells, although accumula- tion of the U85 control intronic scaRNA remained unaltered (Fig. 2D).

To compare the pseudouridylation states of $28 \mathrm{~S}$ rRNA at U3747 and U3749 in the presence and absence of SNORA53, cellular RNAs extracted from HAP1 and SNORA53-KO cells were incubated with CMCT [Ncyclohexyl-N'(2-morpholinoethyl)carbodiimide methop-toluenesulfonate], which efficiently and irreversibly reacts with $\Psi$ (Bakin and Ofengand 1998). The covalently bound bulky CMCT molecule blocks reverse transcriptase elongation $1 \mathrm{nt}$ before the modified $\Psi$ (Fig. 2E). Primer extension analysis of CMCT-treated control HAP1 RNAs with a terminally labeled 28S rRNA-specific oligonucleotide primer detected several known $\Psi$ s, including $\Psi 3737$, $\Psi 3741, \Psi 3742, \Psi 3747$, and $\Psi 3749$ (Fig. 2E, lane 1). In contrast, primer extension mapping of CMCT-modified $28 \mathrm{~S}$ rRNA from SNORA53-KO cells failed to detect pseudouridylation of U3747 and U3749, although synthesis of $\Psi 3737, \Psi 3741$, and $\Psi 3742$ remained unaffected (Fig. 2E, lane 3$)$.

To exclude the formal possibility that disruption of U3747 and U3749 pseudouridylation was a consequence of undesired CRISPR/Cas9 off-target activity, we restored SNORA53 accumulation in the SNORA53-KO cell line by using the pIRESpuro/GL expression plasmid (Fig. 2F; Vitali and Kiss 2019). After selection of a puromycin-resistant monoclonal cell line, accumulation of SNORA53 was confirmed by RNase A/T1 mapping (Fig. 2F, lane 3). Primer extension mapping of $28 \mathrm{~S}$ rRNA pseudouridylation showed that restoration of SNORA53 accumulation re-established pseudouridylation of U3747 and U3749, demonstrating that SNORA53 is responsible for the synthesis of both $\Psi 3747$ and $\Psi 3749$ in the human $28 \mathrm{~S}$ rRNA (Fig. 2E, lane 5).

The 5'-terminal pseudouridylation loop of SNORA53 directs synthesis of both $\Psi 3747$ and $\Psi 3749$ in the $28 S$ rRNA

The 3' hairpin of SNORA53 lacks evolutionarily conserved sequences complementary to rRNAs or other stable cellular RNAs (Supplemental Fig. S1A), suggesting that the elements supporting pseudouridylation of 28SU3747 and 28S-U3749 are located in the $5^{\prime}$-terminal hairpin of SNORA53. To validate this assumption and to learn about the molecular mechanism supporting the predicted double-guide activity of the 5 '-terminal pseudouridylation loop of SNORA53, we performed mutational analysis of SNORA53.

The 5' hairpins of SNORA53 RNAs derived from phylogenetically distant species fold into similar two-dimensional structures composed of a long major hairpin and a short internal stem-loop branched out from the main hairpin (Fig. 3A; Supplemental Fig. S1A). However, upon expression of truncated SNORA53 RNAs lacking either the short internal stem-loop (SNORA53d1) or the distal part of the major 5' hairpin (SNORA53d2) in SNORA53KO cells (Fig. 3A,B), synthesis of both $\Psi 3747$ and $\Psi 3749$ was restored (Fig. 3C, lanes 5,7), indicating that all guide elements supporting U3747 and U3749 pseudouridylation 
A

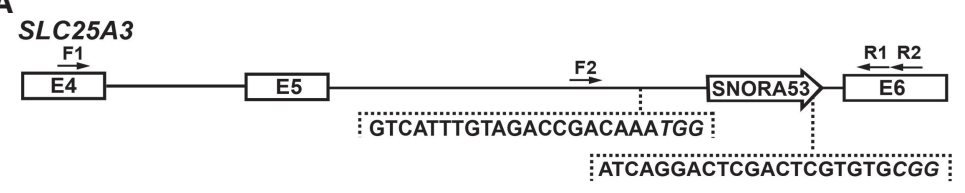

B
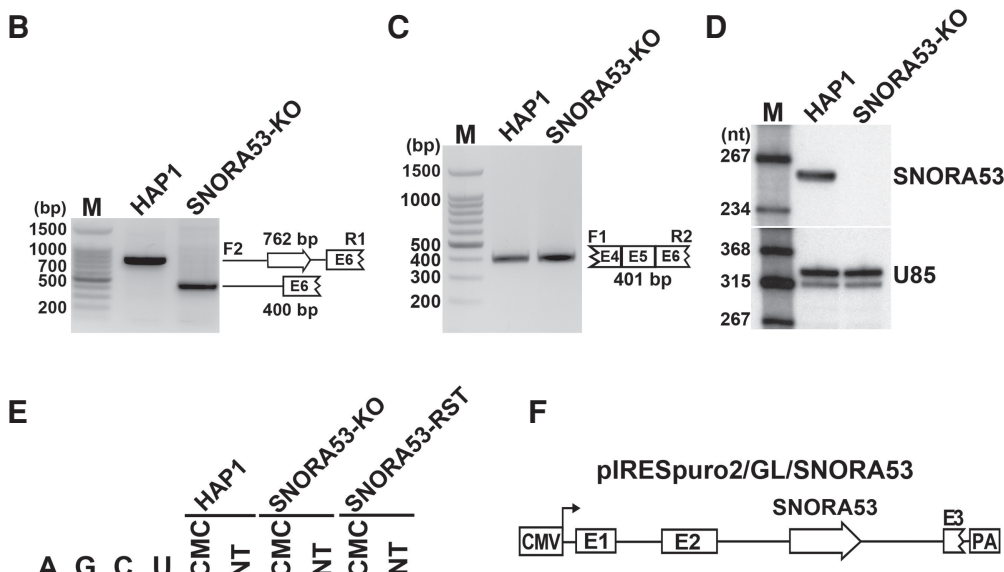

F
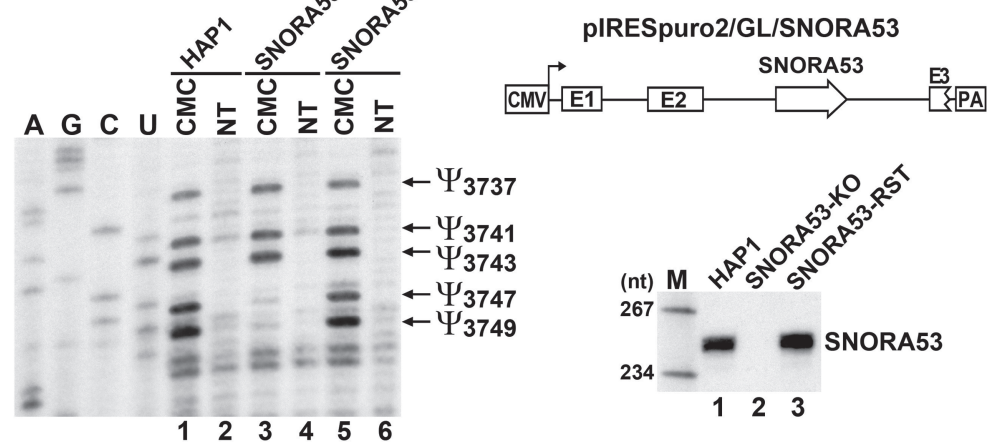

Figure 2. Human SNORA53 is required for synthesis of both $\Psi 3747$ and $\Psi 3749$ in the 28S rRNA. (A) A strategy for CRISPR/Cas9-mediated deletion of human SNORA53 gene in HAP1 cells. Schematic structure of a fragment of the human SLC25A3 gene encompassing exons 4-6 (E4-E6) and the intronic SNORA53 gene (open arrow) is shown. Positions of the forward (F1 and F2) and reverse (R1 and R2) primers used for genomic and RT-PCR analysis are indicated. The genomic sequences targeted by sgRNAs are presented in sense orientation. The PAM sequences are in italics. $(B)$ PCR analysis of genomic DNAs extracted from SNORA53 knockout (SNORA53-KO) and parental HAP1 cells. The amplified DNA fragments were analyzed on $1 \%$ agarose gel. (Lane $M$ ) DNA size markers in base pairs (bp). Schematic structures and expected lengths of the amplified fragments are shown at the right. (C) RT-PCR analysis of the accumulation of spliced SLC25A3 mRNA in SNORA53-KO and HAP1 cells. Structure of the amplified cDNA fragment is shown. $(D)$ SNORA53 accumulation. RNAs isolated from HAP1 and SNORA53KO cells were annealed with internally labeled SNORA53- or U85-specific antisense RNA probes and digested with a mixture of RNase A and T1. The protected RNA fragments were analyzed on a $6 \%$ sequencing gel. (Lane $M$ ) Single-stranded DNA size markers in nucleotides (nt). (E) Mapping of $28 \mathrm{~S}$ rRNA pseudouridylation in HAP1 and SNORA53KO cells before and after restoration of SNORA53 expression (SNORA53-RST). Is covalently modified by CMCT were detected by primer extension analysis using a terminally labeled 28S-specific primer and AMV reverse transcriptase. (Lanes $A, G, C, U$ ) Dideoxy sequencing reactions performed on $28 \mathrm{~S}$ ribosomal DNA with the same $28 \mathrm{~S}$-specific primer. (Lanes NT) Primer extension reactions performed on nontreated control RNAs. $\Psi$ s corresponding to the detected RT stop signals are indicated at the right. $(F)$ Restoration of SNORA53 accumulation in SNORA53-KO cells. Schematic structure of the pIRESpuro2/GL/ SNORA53 expression construct is shown. The coding region of the human SNORA53 gene (open arrow) was inserted into the second intron of the truncated $\beta$-globin gene (E1-E3) placed under the control of the cytomegalovirus (CMV) promoter and the globin polyadenylation signal (PA). The resulting CMV-globin-SNORA53 expression unit was inserted into the pIRESpuro2 expression plasmid and transfected into SNORA53-KO cells. Puromycin-resistant cell colonies were isolated, and expression of SNORA53 in SNORA53-RST cells was verified by RNase A/T1 mapping.

are confined to the proximal part of the SNORA53 5' hairpin. Consistent with its functional importance, the proximal part of the SNORA53 $5^{\prime}$ hairpin contains many phylogenetically conserved nucleotides (Fig. 3A; Supplemental Fig. S1). Significantly, all nucleotides predicted to participate in alternative intra- and intermolecular base-pairing interactions positioning either U3747 or U3749 for pseudouridylation are conserved in the known SNORA53 sequences (Fig. 1C; Supplemental Fig. S1).

To unambiguously demonstrate that the $5^{\prime}$-terminal pseudouridylation loop region of SNORA53 is able to direct synthesis of both 28S- $\Psi 3747$ and 28S- $\Psi 3749$, we attempted to separate the two pseudouridylation guide activities of SNORA53 through construction of mutant SNORA53 RNAs directing either $\Psi 3747$ or $\Psi 3749$ formation. In the proposed SNORA53-28S interactions, in both configuration $\mathrm{A}$ and $\mathrm{B}$, the C10-U13 upstream and the G136-U140 downstream antisense sequences are basepaired with the A3753-G3756 and A3742-C3746 regions of $28 \mathrm{~S}$ rRNA (Fig. 3D, shaded helices). In the center of the guide RNA-substrate RNA three-way helical junction, alternative base-pairing interactions are predicted to govern the structural rearrangements positioning either U3747 or U3749 for pseudouridylation (Fig. 3D, middle panel). Deletion of the 131-CUU-133 bulged nucleotides (CUU133del) was expected to lock the SNORA53-28S rRNA interaction in configuration A and to prevent formation of functional configuration B. Replacement of the 131-CU-132 nucleotides for GA was predicted to stabilize configuration $B$ and to interfere with folding into configuration A. When expressed in SNORA53-KO cells, the SNORA53-CUU133del snoRNP efficiently synthesized $\Psi 3747$, but failed to convert U3749 into $\Psi$ (Fig. 3C, lane 9). On the contrary, the SNORA53CU132GA snoRNP supported $\Psi 3749$ formation, but failed to synthesize $\Psi 3747$ (Fig. 3C, lane 11). Thus, demonstration that sequence alterations can restrict the pseudouridylation guide ability of SNORA53 to the synthesis of either $\Psi 3747$ or $\Psi 3749$ in a predictable way validated the proposed dual guide capacity of the $5^{\prime}$-terminal pseudouridylation loop of SNORA53 and strongly supported the correctness and functional relevance of the 
Jády et al.

A

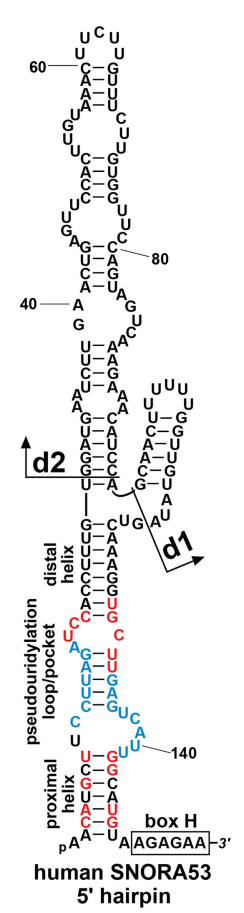

B

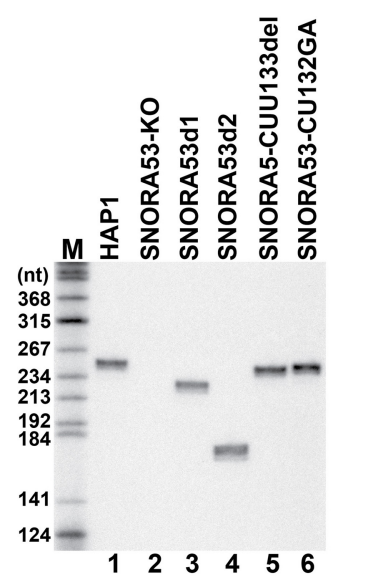

D

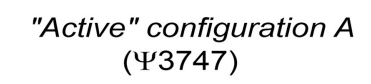

C

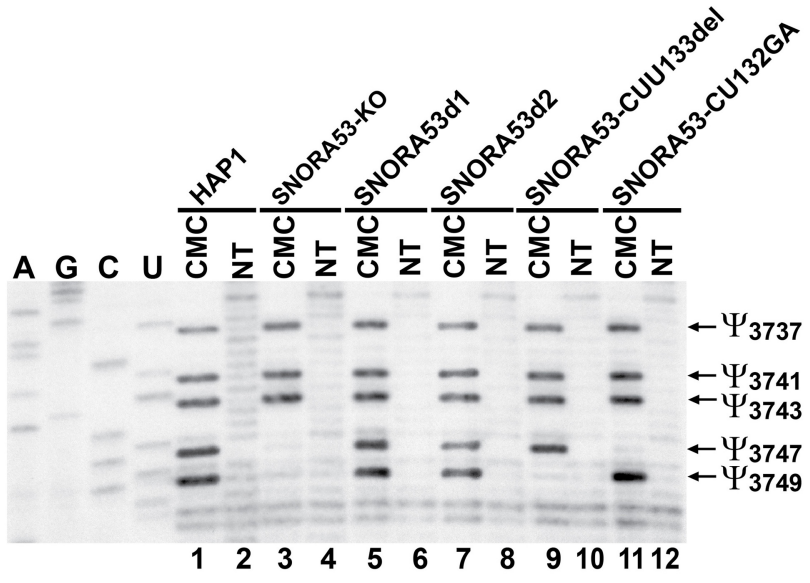

$\begin{array}{lllllllllllll}1 & 2 & 3 & 4 & 5 & 6 & 7 & 8 & 9 & 10 & 1112\end{array}$

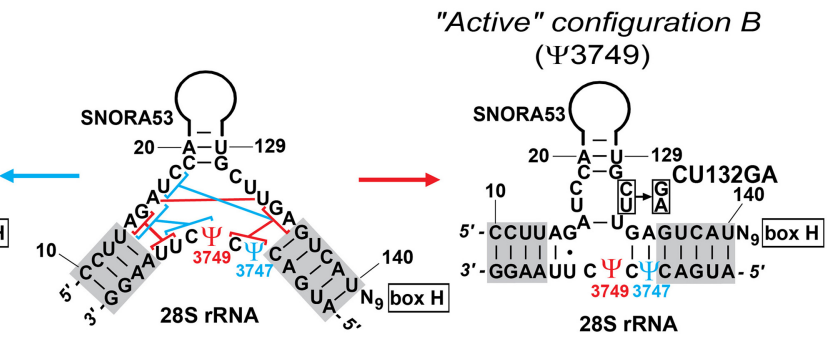

Figure 3. The $5^{\prime}$-terminal pseudouridylation loop of SNORA53 directs pseudouridylation of U3747 and U3749 in the $28 \mathrm{~S}$ rRNA. (A) A computer-predicted secondary structure of the $5^{\prime}$ hairpin of human SNORA53 based on minimum free energy calculation (Zuker 2003). The evolutionarily invariant nucleotides predicted to base-pair with $28 \mathrm{~S}$ rRNA are in blue. Other conserved nucleotides are in red. Arrows indicate internal deletions $\mathrm{d} 1$ and $\mathrm{d} 2$. (B) Expression of mutant SNORA53 RNAs in SNORA53-KO cells monitored by RNase A/T1 protection with sequence-specific antisense RNA probes. As controls, accumulation of endogenous SNORA53 was also tested in HAP1 and SNORA53-KO cells. (Lane $M$ ) Single-stranded DNA size markers. (C) Primer extension mapping of 28S rRNA pseudouridylation in HAP1 and SNORA53-KO cells either lacking or expressing mutant SNORA53 RNAs as indicated above the lanes. For details, see the legend for Figure 2E. $(D)$ Proposed structural rearrangements of the three-way helical junctions formed by the $5^{\prime}$-terminal pseudouridylation loop of human SNORA53 and 28S rRNA positioning either U3747 or U3749 for pseudouridylation. The proposed alternative base-pairing interactions leading to "active" configuration A or B are indicated by blue and red lines, respectively. The common base-pairing interactions present in both configurations are shaded. The deleted or altered nucleotides in mutant SNORA53-CUU133del and SNORA53-CU132GA RNAs are boxed.

proposed alternative base-pairing interactions of SNORA53 with 28S rRNA positioning U3747 or U3749 for pseudouridylation.

The bulged ribosomal U3752 residue supports the dual guide activity of SNORA53

Normally, the antisense elements of pseudouridylation guide RNAs form perfect double helices with their target sequences (Fig. 1B; Ganot et al. 1997a). However, in configuration A positioning U3747 for pseudouridylation, the upstream antisense target stem (UATS) formed by the upstream antisense element of SNORA53 (10CCUUAGA-17) and its 28S rRNA target sequence (3749-UCUUAAGG-3756) is interrupted by the bulged U3752 ribosomal residue (Figs. 1C, 3D). Given that the SNORA53 antisense and the $28 \mathrm{~S}$ target sequences are conserved, we speculated that bulging of 28S-U3752 may contribute to the dual guide capacity of the 5 -terminal pseudouridylation loop of SNORA53. To test this hypothesis, an extra A residue was inserted into SNORA53 between U13 and A14 in order to base-pair with the bulged ribosomal U3452 residue (Fig. 4A). Expression of the mutant SNORA53-U13+A RNA in SNOR53-KO cells restored pseudouridylation of U3747, but failed to support formation of $\Psi 3749$ (Fig. 4A, lane 5).

Next, we tested the in vivo pseudouridylation of $28 \mathrm{~S}$ rRNA sequences lacking U3752. Small fragments of the human 28S rRNA from position G3729 to G3759 either containing (28S-WT) or lacking (28S-U3752del) U3752 were transiently expressed in mouse cells by using the $\mathrm{pW}(\mathrm{Xb} / \mathrm{Xh})$ mouse ribosomal minigene expression plasmid (Fig. 4B; Hadjiolova et al. 1994). Since the ribosomal minigene is transcribed by RNA polymerase I in the nucleolus, rRNA sequences imbedded within the nascent minigene transcript can undergo snoRNP-mediated modifications (Ganot et al. 1997a). Primer extension analysis after CMCT treatment clearly demonstrated that synthesis of $\Psi 3749$ was largely inhibited or fully abolished in the 28S-U3752del transcript (Fig. 4B, lane 3), but it was unaffected in the control 28S-WT RNA (Fig. 4B, lane 1). Importantly, both 28S-U3752del and 28S-WT RNAs were 
A
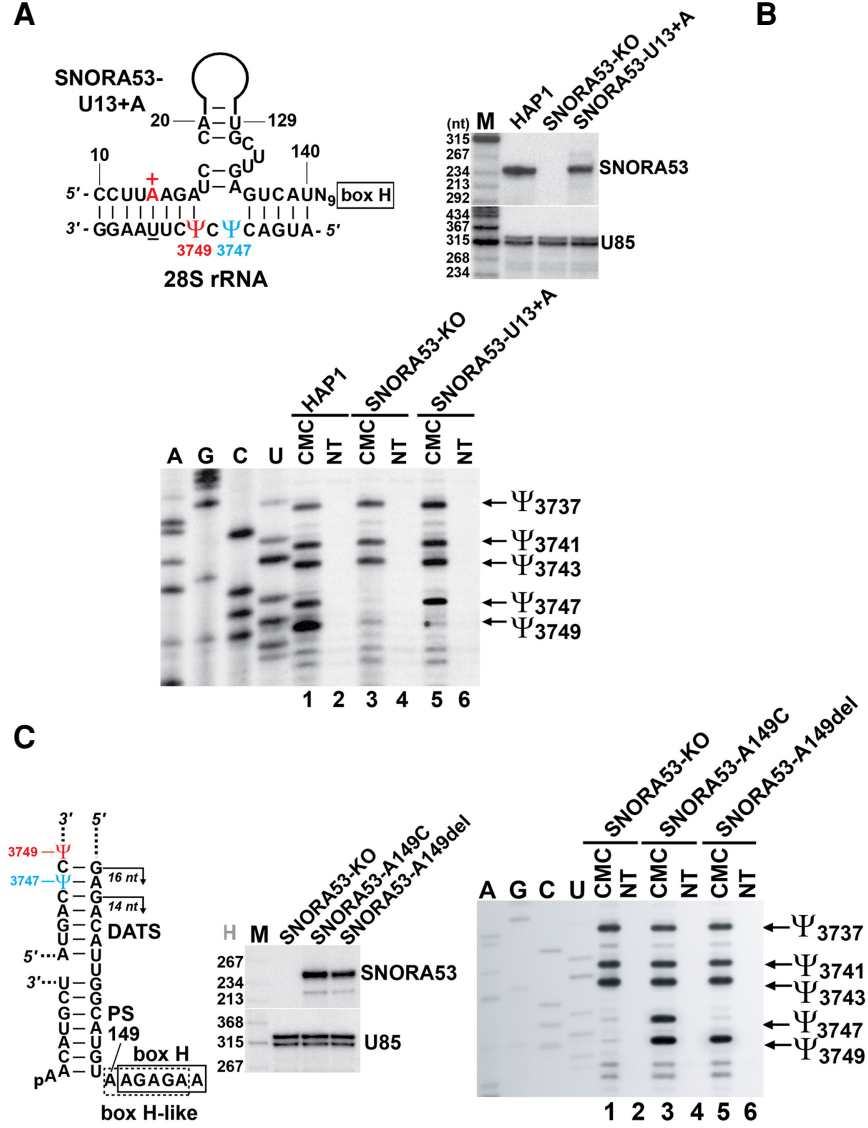

123456
B
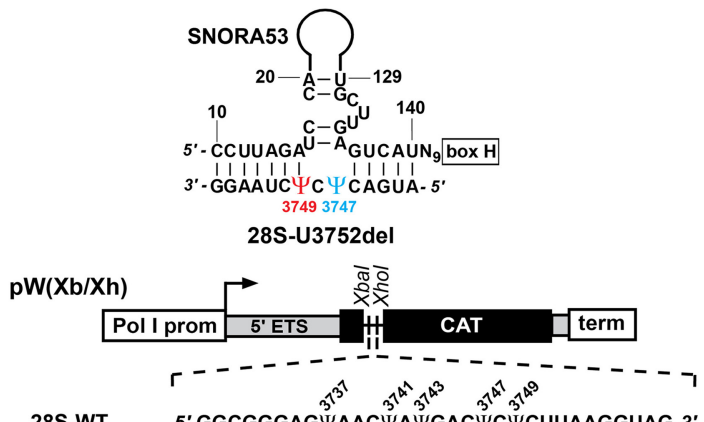

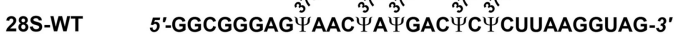

28S-U3752del 5'-GGCGGGAG $\Psi A A C \Psi A \Psi G A C \Psi C \Psi C U-A A G G U A G-3$ '

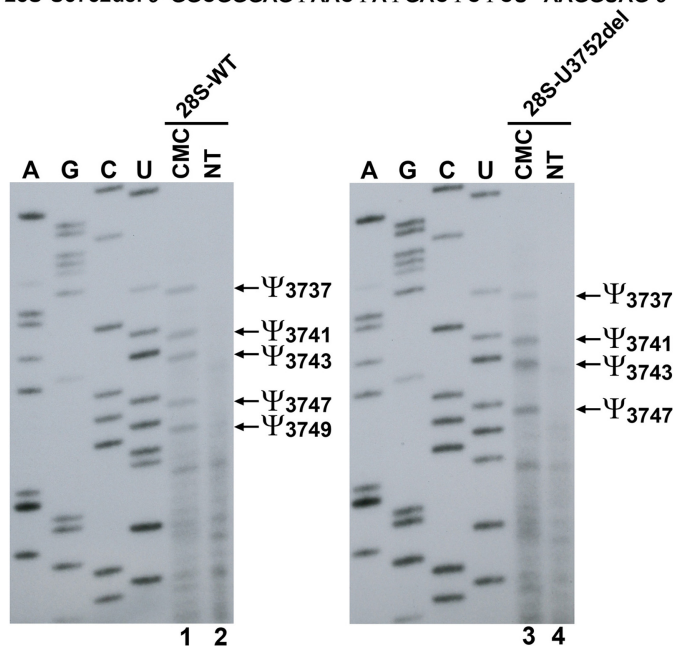

Figure 4. Structural requirements of the dual pseudouridylation guide activity of the $5^{\prime}$ hairpin of SNORA53. (A) In vivo pseudouridylation of 28S rRNA with a mutant SNORA53 RNA. (Top left panel) A predicted interaction of endogenous $28 \mathrm{~S}$ rRNA with an ectopically expressed mutant SNORA53 RNA carrying an extra A (A+, in red) inserted between U13 and A14. The 28S-U3752 residue is underlined. Please note that 28S-U3751 could also bulge out in configuration A, but the upstream antisense element of SNORA53-U13+A is able to base-pair with both U3751 and U3752. Accumulation of SNORA53-U13+A in SNORA53-KO cells was verified by RNase mapping. As controls, accumulation of endogenous SNORA53 and U85 RNAs was also tested. (Bottom panel) Pseudouridylation of 28S rRNA was monitored by primer extension mapping in HAP1 and SNORA53-KO cells lacking or expressing SNORA53-U13+A. For other details, see the legend for Figure 2E. (B) In vivo pseudouridylation of transiently expressed mutant 28S rRNA (28S-U3752del) sequences lacking U3752 in mouse 3T3 cells. (Top panel) A predicted interaction of endogenous mouse SNORA53 with 28S-U3752del rRNA and schematic structure of the mouse $\mathrm{pW}(\mathrm{Xb} / \mathrm{Xh})$ expression construct are shown. The mouse RNA polymerase I promoter (Pol I prom) and terminator (term), the $5^{\prime}$ - and $3^{\prime}$-terminal regions of the $5^{\prime}$ and $3^{\prime}$ external transcribed sequences (ETS; gray boxes), and a fragment of the chloramphenicol acetyltransferase (CAT) gene are indicated (Hadjiolova et al. 1994). The nucleotide sequences of wild-type (28S-WT) and mutant (28SU3752del) 28S rRNA fragments with the known ribosomal $\Psi$ s are shown. (Bottom panel) Pseudouridylation of the expressed 28S-WT and 28S-U3752del ribosomal minigene transcripts was monitored by primer extension analysis. $(C)$ Correct positioning of the $\mathrm{H}$ box relative to the selected substrate Us is essential for the dual guide activity of the $5^{\prime}$ hairpin of SNORA53. (Left panel) Interaction of the descending strand of the $5^{\prime}$ hairpin of SNORA53 with 28S rRNA positioning U3747 and U3749 for pseudouridylation. A putative H-box-like motif is highlighted in the dashed box. The A149 residue was deleted in SNORA53-A149del and replaced for C in SNORA53-A149C. (Middle panel) Accumulation of ectopically expressed SNORA53-A149C and SNORA53-A149del RNAs and endogenous U85 scaRNA in SNORA53KO cells was measured by RNase mapping. (Right panel) Primer extension mapping of 28S rRNA pseudouridylation in SNORA53-KO cells lacking or expressing SNORA53-A149C or SNORA53-A149del RNAs.

faithfully pseudouridylated at $\Psi 3747$ and other known pseudouridylation sites: $\Psi 3737, \Psi 3741$, and $\Psi 3743$. Thus, stabilization of the upstream antisense target stem (UATS) of SNORA53 in configuration A, either by removal of the bulged U3752 ribosomal residue or by insertion of a complementary A residue into the upstream antisense sequence of SNORA53, promoted synthesis of $\Psi 3747$ and prevented formation of $\Psi 3749$, suggesting that accurate function of the dual guide loops necessitates comparable thermodynamic stabilities of the alternative antisense target base-pairing interactions.

In most $\mathrm{H} / \mathrm{ACA}$ guide RNA-substrate RNA interactions, the selected target $\mathrm{Us}$ are separated from the $\mathrm{H}$ or ACA boxes by 14- to 15-nt-long guide RNA sequences (Supplemental Fig. S2). In the proposed alternative SNORA53-28S rRNA interactions, U3747 is positioned at the optimal 14-nt distance from the H box, but U3749 is separated by $16 \mathrm{nt}$, which is a less common 
configuration (Fig. 4C, left panel). Besides the canonical H box (150-AGAGAA-155), SNORA53 contains another overlapping H-box-like sequence (149-AAGAGA-154) that is separated only by $15 \mathrm{nt}$ from U3749. However, disruption of the potential dyskerin binding capacity of the H-like motif through conversion of A149 into C had no effect on the synthesis of 28S- $\Psi 3747$ and 28S- $\Psi 3749$, suggesting that dyskerin bound to the canonical $\mathrm{H}$ box of SNORA53 can convert both U3747 and U3749 into $\Psi$ (Fig. 4C, lane 3). More unexpectedly, deletion of A149 that reduced the distance between the $\mathrm{H}$ box of SNORA53-A149del and the U3747 target residue from 14 to $13 \mathrm{nt}$ fully abolished pseudouridylation of U3747 (Fig. 4C, lane 5), contrary to the fact that 13-nt-long spacers separating target Us from the $\mathrm{H}$ or ACA boxes are relatively frequent (Supplemental Fig. S2). In addition to the $\mathrm{H}$ and ACA motifs, Cbf5/dyskerin also interacts with the minor grove of the proximal stem (PS) and the downstream antisense target stem (DATS) through its PUA (pseudouridine synthase and archaeosine transglycosylase) and catalytic domains, respectively (Fig. 1B). The PS-DATS joint helical stack seems to serve as a molecular ruler measuring the distance between the target Us and $\mathrm{H} /$ ACA boxes. In the SNORA53-28S rRNA interaction, the PS and DATS helices are separated by only one unpaired loop nucleotide (U141) (Fig. 4C, left panel). This may limit the structural plasticity of the PS-DATS helical stack of SNORD53-A149del required for correct positioning of U3747 for pseudouridylation.

\section{Human H/ACA pseudouridylation loops with potential dual guide capacities}

To identify additional human H/ACA pseudouridylation loops with potential dual guide capacities, we collected the human spliceosomal and ribosomal $\Psi$ s that are located next to each other $(\Psi \Psi)$ or are separated by $1 \mathrm{nt}(\Psi \mathrm{N} \Psi)$ and listed them together with the corresponding H/ACA hairpins assigned for their site-specific selection (Fig. 5A; Lestrade and Weber 2006; Jorjani et al. 2016; Taoka et al. 2018). This compilation confirmed that most $\Psi$ s, even in densely pseudouridylated regions, are selected individually by dedicated pseudouridylation loops. Nevertheless, inspection of the listed H/ACA hairpins revealed that the $3^{\prime}$-terminal pseudouridylation loops of SCARNA14, SCARNA8, and SCARNA1, which had been earlier assigned for pseudouridylation of the U2 snRNA at $\Psi 7, \Psi 44$, and $\Psi 89$, respectively (Darzacq et al. 2002; Kiss et al. 2004; Schattner et al. 2006), might also direct synthesis of the neighboring "orphan" $\Psi 6, \Psi 43$, and
A

\begin{tabular}{|c|c|}
\hline \multicolumn{2}{|l|}{ U1 snRNA } \\
\hline $5-\Psi \Psi-6$ & SCARNA16(3'hp); SCARNA18(3'hp) \\
\hline \multicolumn{2}{|l|}{ U2 snRNA } \\
\hline $6-\Psi \Psi-7$ & SCARNA14(3'hp); SCARNA14(3'hp) \\
\hline \multirow[t]{2}{*}{ 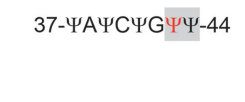 } & SCARNA15(3'hp); SCARNA4(3'hp); \\
\hline & $\begin{array}{l}\text { SCARNA4(5'hp); SCARNA8(3'hp); } \\
\text { SCARNA8(3'hp) }\end{array}$ \\
\hline 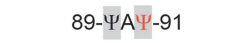 & SCARNA1(3'hp); SCARNA1(3'hp) \\
\hline \multicolumn{2}{|l|}{ 18S rRNA } \\
\hline $34-\Psi C \Psi-36$ & SNORA50(5'hp); SNORA69(3'hp) \\
\hline $649-\Psi A \Psi-651$ & SNORA46(5'hp); SNORA20(5'hp) \\
\hline $814-\Psi \Psi-815$ & SNORA77(5'hp); SNORA28(5'hp) \\
\hline $1045-\Psi \Psi-1046$ & SNORA57(3'hp); SNORA57(3'hp) \\
\hline \multicolumn{2}{|l|}{ 28S rRNA } \\
\hline 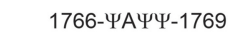 & ?; ?; SNORA9(3'hp) \\
\hline $1847-\Psi \cup \Psi-1849$ & SNORA32(5'hp); SNORA51(5'hp) \\
\hline 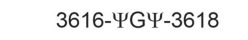 & SNORA6(5'hp); SNORA19(5'hp) \\
\hline 3741-ЧАЧ-3743 & SNORA74(5'hp); SNORA74(3'hp) \\
\hline $3747-\Psi C \Psi-3749$ & SNORA53(5'hp), SNORA53(5'hp) \\
\hline 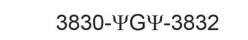 & SNORA62(5'hp); SNORA8(3'hp) \\
\hline
\end{tabular}

B

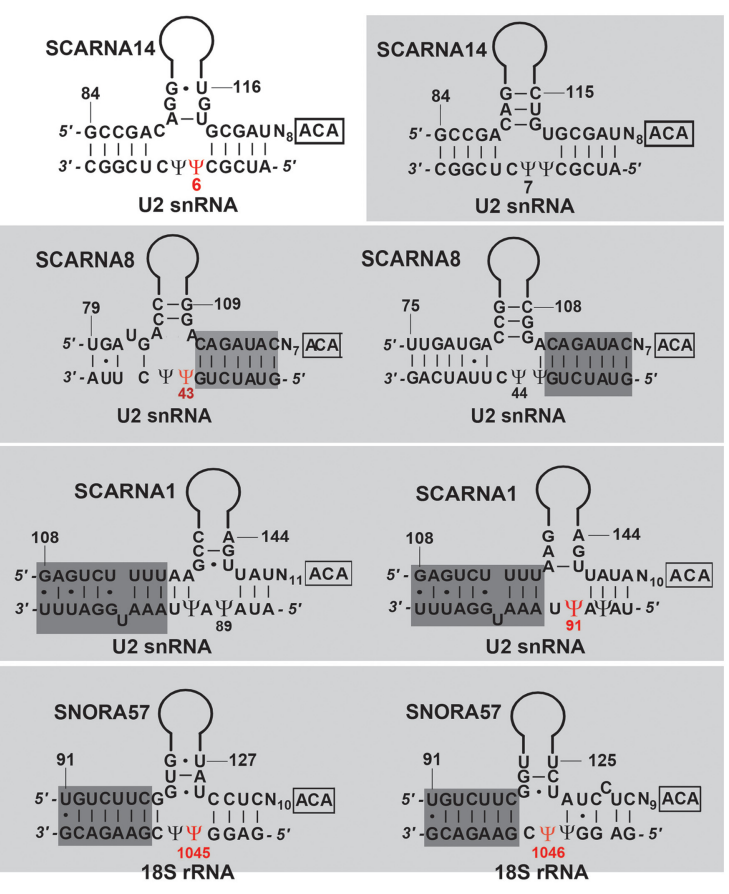

Figure 5. Human pseudouridylation guide loops with possible dual guide capacities. (A) Closely located human spliceosomal and ribosomal $\Psi$ s are listed together with the corresponding pseudouridylation guide RNA hairpins (5'hp and 3'hp) connected to their synthesis. Hairpins newly implicated in dual pseudouridylation reactions and their predicted target $\Psi$ s are highlighted in red. Participation of the shaded H/ACA hairpins in the synthesis of their predicted target $\Psi$ s has been experimentally validated in this study. $(B)$ Potential alternative base-pairing interactions of H/ACA pseudouridylation guide loops with their target RNAs positioning consecutive ribosomal and spliceosomal Us for pseudouridylation. Direction of the synthesis of $\Psi 7, \Psi 44$, and $\Psi 89$ in the U2 snRNA by human SCARNA14, SCARNA8, and SCARNA1, respectively, has been proposed earlier (Darzacq et al. 2002; Kiss et al. 2004; Schattner et al. 2006). The newly predicted target $\Psi$ s are highlighted in red. The functional interactions shaded in light gray have been experimentally validated in this work. Base-pairing interactions common in the alternative functional configurations are shaded in dark gray. For the interaction of SNORA53 and 28S rRNA, see Figure 3D. 
$\Psi 91$ residues through formation of alternative base-pairing interactions (Fig. 5B). In fact, the $3^{\prime}$ hairpin of the Xenopus equivalent of human SCARNA8, pugU2-34/44, has already been reported to direct pseudouridylation of the U2 snRNA at both U43 and U44 (Deryusheva and Gall 2013). Finally, re-examination of human orphan H/ACA RNAs revealed that the $3^{\prime}$ hairpin of SNORA57 carries a putative pseudouridylation loop that, in principle, could direct synthesis of both $\Psi 1045$ and $\Psi 1046$ in the $18 \mathrm{~S}$ rRNA. Thus, our search identified four novel putative dual pseudouridylation guide loops amenable for experimental testing.

The 3' hairpins of SCARNA1, SCARNA8, and SNORA57 direct synthesis of two adjacent $\Psi$ s in the U2 and $18 S$ RNAs

To examine the in vivo pseudouridylation guide competences of SCARNA1, SCARNA8, SCARNA14, and SNORA57, the single-copy genes of these intronic RNAs were deleted with CRISPR-Cas9 genome editing in human HAP1 cells (Supplemental Fig. S3). Disruption of the accumulation of each target H/ACA RNA in the CRISPR-treated KO cells was confirmed by RNase A/T1 mapping (Fig. 6A), and pseudouridylation of the predicted target Us in U2 and 18S RNAs was monitored by primer extension analysis (Fig. 6B). Abrogation of SCARNA1 and SCARNA8 accumulation fully obliterated pseudouridylation of the U2 snRNA at the U89/U91 and U43/U44 residues, respectively. Lacking U2- $\Psi 43$ formation in the absence of SCARNA8 was somewhat unexpected, because formation of Xenopus U2- $\Psi 43$ had been reported to be a redundant reaction implicating both SCARNA8 and a stand-alone $\Psi$ synthase, Pus1 (Deryusheva and Gall 2017). Disruption of SNORA57 accumulation abolished $\Psi 1045$ and $\Psi 1046$ synthesis in the 18S rRNA. Importantly, restoration of SCARNA1, SCARNA8, and SNORA57 accumulations in the corresponding KO cells re-established synthesis of U2- $\Psi 89 / \Psi 91$, U2- $\Psi 43 / \Psi 44$, and $18 S-\Psi 1045 / \Psi 1046$, corroborating the notion that the $3^{\prime}$-terminal pseudouridylation loops of SCARNA1, SCARNA8, and SNORA57 possess dual guide activities. Finally, disruption of SCARNA14 expression demolished formation of $\Psi 7$ in the human U2 snRNA, but it had no impact on the synthesis of $\Psi 6$, excluding the proposed dual guide capacity of the $3^{\prime}$ hairpin of SCARNA14 and confirming the idea that it directs synthesis of U2- 47 (Schattner et al. 2006).

\section{Alternative base-pairings with the H/ACA antisense sequences are the major determinants of dual guide activities}

We have already demonstrated above that the dual pseudouridylation guide activity of the $5^{\prime}$ hairpin of SNORA53 can be confined to the synthesis of one $\Psi$ through structural manipulation of its DS region (Fig. 3). These results seem to support the functional importance of the DS regions of dual pseudouridylation loops. The tested CUU133del and CU132GA mutations of SNORA53 (Fig.
A
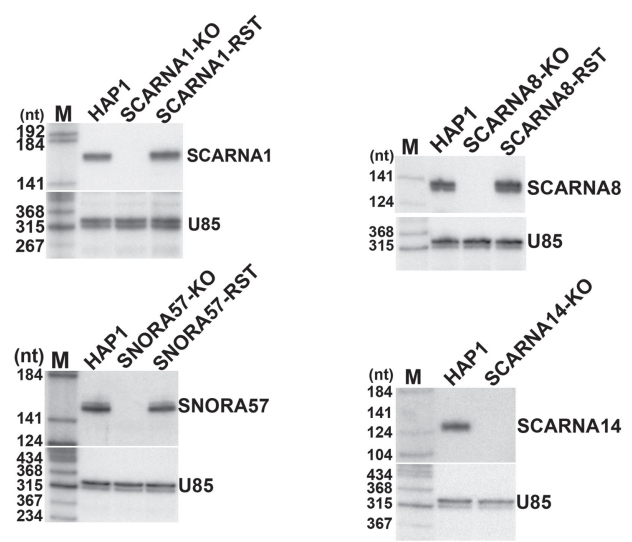

B
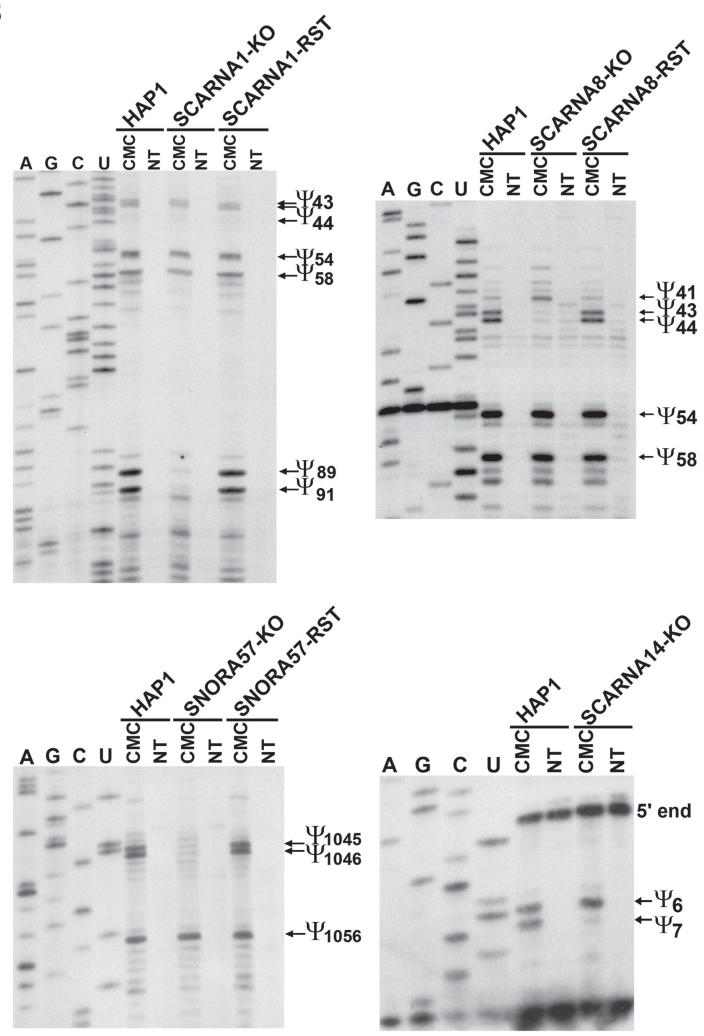

Figure 6. The 3'-terminal pseudouridylation loops of human SCARNA1, SNORA57, and SCARNA8 possess dual guide capacities. (A) Expression of SCARNA1, SNORA57, SCARNA8, and SCARNA14 measured by RNase mappings. After deletion of the SCARNA1, SNORA57, SCARNA8, and SCARNA14 genes in HAP1 cells (Supplemental Fig. S3), each KO cell line was transformed by pIRESpuro/GL plasmids expressing SCARNA1, SCARNA8, or SNORA57 (RST cells). (B) Primer extension mapping of U2 snRNA and 18S rRNA pseudouridylation in the absence and presence of SCARNA1, SCARNA8, SNORA57, and SCARNA14 as indicated above the lanes. For other details, see the legend for Figure 2E.

3D), while promoting formation of one of the two alternative active configurations of SNORA53, prevented formation of the other one. To further understand the 
importance of the DS region in the activities of H/ACA dual pseudouridylation guide loops, a series of mutant SNORA57 and SCARNA1 RNAs were expressed in SNORA57-KO or SCARNA1-KO cells, and their pseudouridylation guide capacities were determined (Fig. 7).

Through increasing the stability of the DS regions, the 124-UUC-126 deletion and the U125-to-A conversion in SNORA57 were predicted to promote formation of active configuration A or B and synthesis of $18 \mathrm{~S}-\Psi 1045$ or $18 \mathrm{~S}$ $\Psi 1046$, respectively (Fig. 7A). However, while the 124UUC-126 deletion was predicted to interfere with formation of active configuration B, the U125A mutation of SNORA57 did not exclude the formal possibility of folding into active configuration $\mathrm{A}$. Upon expression in SNORA57-KO cells (Fig. 7B), the SNORA57-UUC126del RNA proved to be efficient in $18 \mathrm{~S}-\Psi 1045$ synthesis, but failed to govern 18S- 1046 formation (Fig. 7B, lane 5). In contrast, the SNORNA57-U125A RNA retained its dual guide ability to direct synthesis of both $\Psi 1045$ and 世1046 (Fig. 7B, lane 7).

Next, we further assessed the contribution of the distal stem (DS) region to the dual pseudouridylation guide activity of the $3^{\prime}$ hairpin of SCARNA1. A series of mutant SCARNA1 RNAs carrying nucleotide alterations slightly increasing or reducing the stability of the DS region were expressed in SCARNA1-KO cells (Fig. 7C). Importantly, none of the tested mutations were expected to fully exclude folding into any of the alternative active configurations of the 3' hairpin of SCARNA1. The A144-to-G transition in SCARNA1-A144G was expected to promote DS stability in configuration A directing U2-489 synthesis and to slightly destabilize the DS region in configuration B governing U2- $\Psi 91$ synthesis. In contrast, the G145-to-U (G145U), the 144-AG-145-to-CU (AG145CU), and the C121-to-G (C121G) base conversions in the DS region of SCARNA1 were predicted to support formation of configuration $B$ and to disfavor folding into configuration A. Insertion of AG dinucleotides between the C122 and A123 residues of SCARNA1 $(\mathrm{C} 122+\mathrm{AG})$ was predicted to increase the stem stability in both configurations A and B, but leaving six unpaired nucleotides highly destabilizing the proximal part of the DS region in configuration B. Finally, replacement of U147 for a C residue was predicted to disrupt the A117:U146 base-pairing interaction closing the pseudouridylation loop in configuration B. However, when expressed in SCARNA1-KO cells (Fig. 7D, top panel), the mutant SCARNA1 RNAs retained dual guide activity directing efficient synthesis of both $\Psi 89$ and $\Psi 91$ in the U2 snRNA (Fig. 7D, bottom panel), confirming that structural alterations in the distal stem region, if they do not exclude formation of one functional configuration, have no significant impact on the dual pseudouridylation guide capacity of the $3^{\prime}$-terminal guide loop of SCARNA1. These results, together with the observations that minor structural alterations in the upstream antisense target stem (UATS) could completely abolish the dual guide activity of the $5^{\prime}$ hairpin of SNORA53 (Fig. 4), suggest that the alternative base-pairing interactions formed between the antisense sequences and the target RNA are the major determinants of the activity of dual pseudouridylation guide loops.

\section{Discussion}

Post-transcriptional pseudouridylation of eukaryotic spliceosomal snRNAs and rRNAs is essential for efficient and correct pre-mRNA splicing and protein synthesis. Site-specific synthesis of $\sim 130$ Is in human snRNAs and rRNAs is achieved mostly by H/ACA pseudouridylation guide RNPs. In contrast to stand-alone $\Psi$ synthases that recognize local RNA structures and frequently synthesize several closely placed $\Psi$ s (Spenkuch et al. 2014), the H/ACA RNPs have been believed to direct synthesis of a single $\Psi$ in their target sequences. However, here we have demonstrated that the pseudouridylation guide loops in the $5^{\prime}$-terminal hairpin of human SNORA53 and in the $3^{\prime}$ hairpins of human SNORA57, SCARNA1, and SCARNA8 H/ACA RNAs are able to support synthesis of two consecutive $\Psi \mathrm{s}(\Psi \Psi$ or $\Psi N \Psi)$ through formation of alternative base-pairing interactions with their target RNAs, breaking with the notion that pseudouridylation guide RNPs lack site promiscuity. Apparently, synthesis of two $\Psi$ s is not a consequence of defective or inaccurate functions of the dual guide loops of human SNORA53, SNORA57, SCARNA1, and SCARNA8, because their substrate Us are pseudouridylated with practically 100\% efficiency (Reddy and Busch 1988; Taoka et al. 2018). Even more telling, all guide RNA nucleotides implicated in inter- and intramolecular basepairings promoting alternative target RNA interactions are perfectly conserved during evolution (Supplemental Figs. S1, S4). These strong evolutionary constraints clearly demonstrate the functional importance of these nucleotides in correct and efficient dual pseudouridylation reactions.

In principle, synthesis of two $\Psi$ s by dual pseudouridylation guide loops could be accomplished through one or two independent target binding events, depending on whether the guide RNP dissociates from or remains associated with its target RNA after the first pseudouridylation reaction. Although we cannot unambiguously distinguish between these two possibilities, we prefer the "one binding-two pseudouridylation" model. Continuous guide RNA-target RNA association could increase the efficiency of the two subsequent pseudouridylation reactions directed by the same guide loop. In this context, it is noteworthy that the proposed alternative guide substrate configurations always share common interacting core domains that could continuously anchor the guide RNP to the target RNA without interfering with the structural rearrangements supporting the two pseudouridylation reactions (Figs. 3D, 5B, shaded helices). In the "two-binding model," after dissociation of the guide RNP following the first pseudouridylation reaction, the dual pseudouridylation loop region of the released guide RNP is expected to undergo structural rearrangements to conform to the second pseudouridylation reaction. Such conformational changes in the guide RNA could 
A

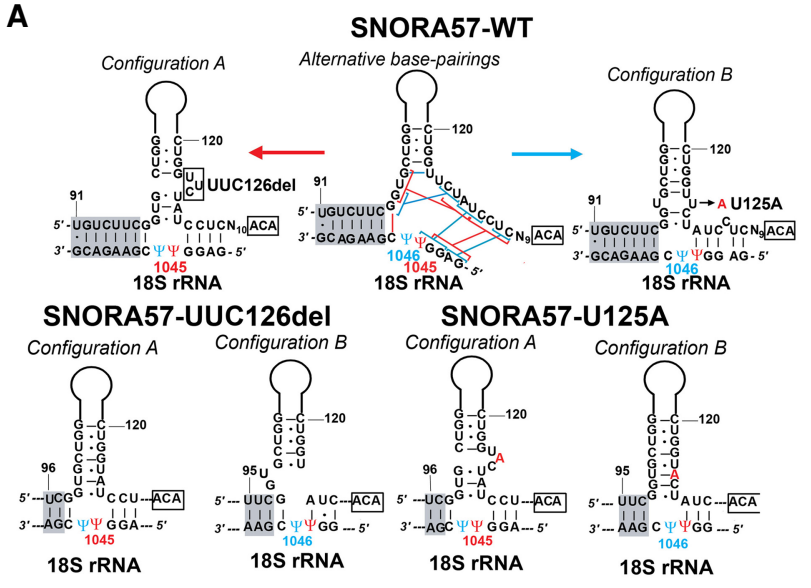

C
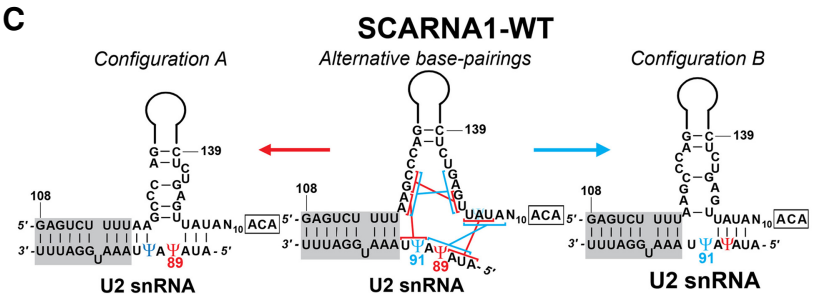

SCARNA1-G145U

Configuration $A$ Configuration $B$

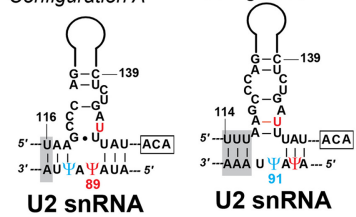

U2 snRNA

U2 SnRNA
B

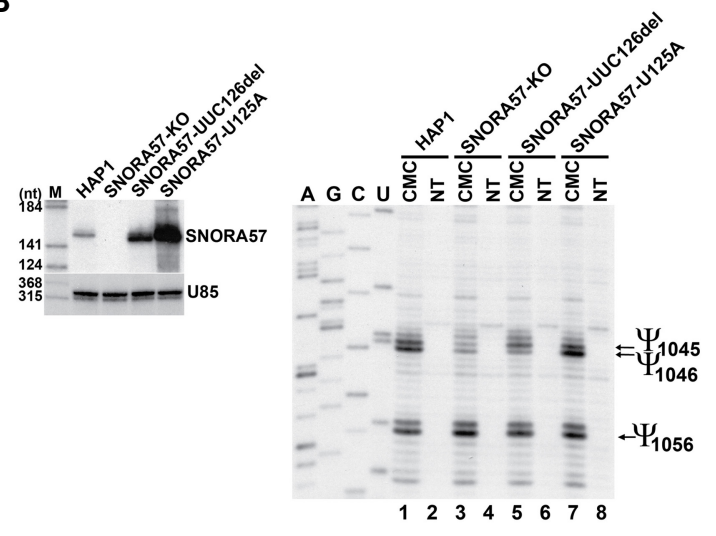

D
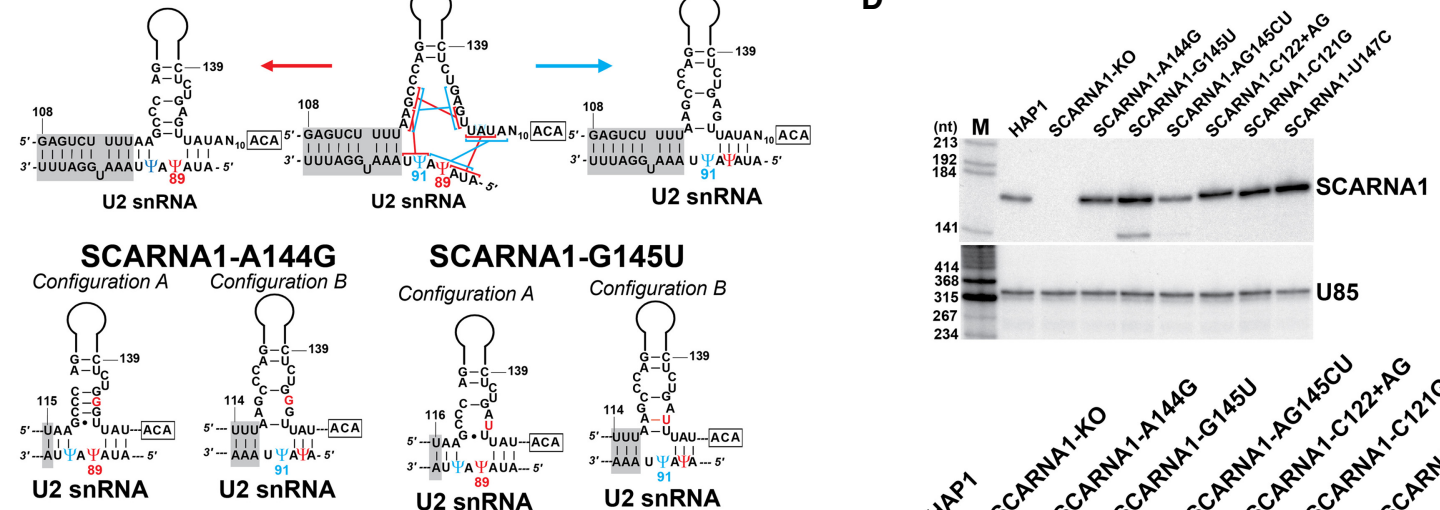

SCARNA1-AG145CU

Configuration A Configuration B

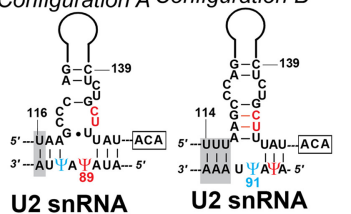

SCARNA1-C122+AG

Configuration $A$ Configuration $B$

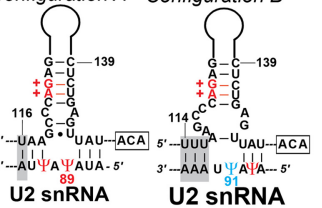

SCARNA1-C121G

Configuration A Configuration $B$

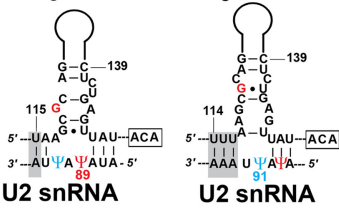

SCARNA1-U147C

Configuration $A$ Configuration $B$

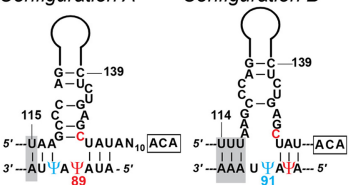

U2 snRNA

U2 SnRNA

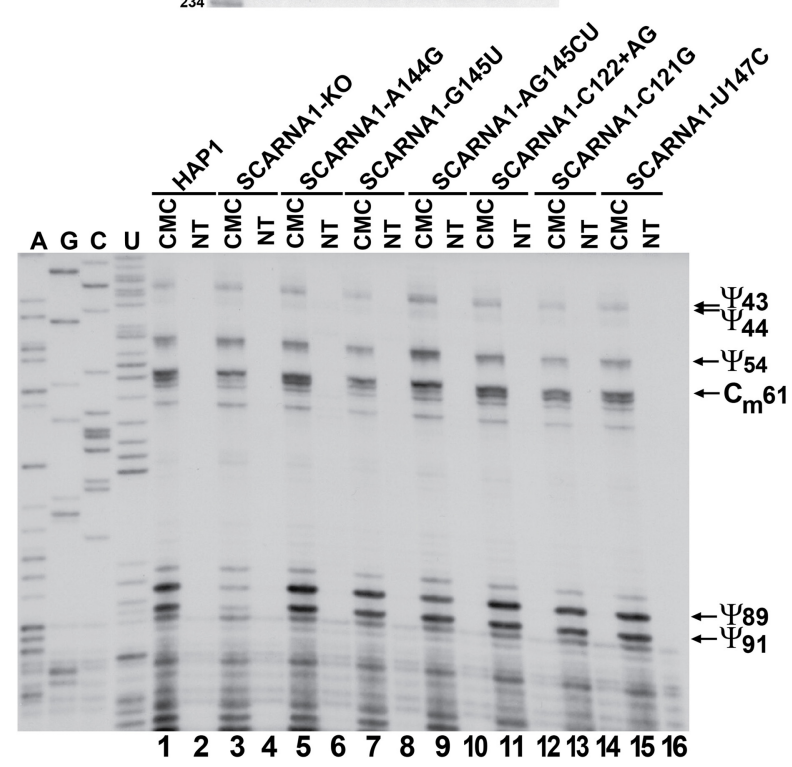

Figure 7. Mutational analyses of SNORA57 and SCARNA1. (A) Predicted base-pairing interactions of wild-type (WT) and mutant SNORA57 RNAs with 28S rRNA in configurations A and B directing synthesis of $\Psi 1045$ and $\Psi 1046$, respectively. (B) Accumulation of mutant SNORA57 RNAs in SNORA57-KO cells and pseudouridylation of endogenous 28S rRNA. (Left panel) Accumulation of SNORA57-UUC126del and SNORA57-U125A RNAs was detected by RNase protection with sequence-specific probes. (Right panel) The pseudouridylation state of $18 \mathrm{~S}$ rRNA in wild-type HAP1 and SNORA57-KO cells lacking or expressing the indicated mutant SNORA57 RNAs was measured by primer extension. $(C)$ Predicted interactions of wild-type and mutant SCARNA1 RNAs with U2 snRNA directing pseudouridylation of U89 (configuration A) or U91 (configurations B). The nucleotide alterations in the mutant SCARNA1 RNAs are highlighted in red. $(D)$ In vivo pseudouridylation activity of mutant SCARNA1 RNAs. (Top panel) Expression of mutant SCARNA1 RNAs in SCARNA1-KO cells was monitored by RNase protection. The lower band in the SCARNA1-G145U lane likely represents a degradation product of SCARNA1-G145U. (Bottom panel) Primer extension mapping of U2 snRNA pseudouridylation at U89 and U91 in the absence (SCARNA1-KO) or presence of wild-type (HAP1) and mutant SCARNA1 RNAs as indicated above the lanes. For other details, see the legend for Figure 2E. 
be governed by the distal stem region through slightly altering and repositioning the single-stranded antisense sequences. However, the dual guide loops of SNORA53, SNORA57, and SCARNA1 are topped by loosely structured and thermodynamically unstable distal stem regions, arguing against a role in shaping their pseudouridylation loops (Figs. 3D, 5B). In line with this conclusion, nucleotide alterations in the distal stem region had no significant impact on the dual pseudouridylation capacity of the 3 -terminal guide loops of SCARNA1 and SNORA57 (Fig. 7). Instead, our data supported the idea that correct remodeling of the target RNA-guide RNA three-way helical junctions is dictated predominantly by formation of alternative antisense target interactions (Fig. 7).

Dissection of the molecular mechanisms driving the appropriate guide RNA-target RNA structural rearrangements supporting the correct and efficient function of dual pseudouridylation guide RNPs remains a challenge for the future. In the secondary structures of H/ACA RNAs defined by minimal free energy calculation, the pseudouridylation loops frequently fold into partially closed structures (see Fig. 3A; Supplemental Fig. S1; data not shown), suggesting that the associated H/ACA proteins contribute to the correct folding of functionally competent pseudouridylation loops. Indeed, the catalytic surface of Cbf5 loosely and nonspecifically associates with the pseudouridylation loop regions of H/ACA RNAs (Li and Ye 2006). In the substrate-bound H/ACA $\mathrm{RNP}$, the conserved arginine-rich thumb region of Cbf5 interacts with the substrate RNA locking the target $U$ at the active site of the RNP (Duan et al. 2009). Garl interacts with the catalytic domain of archaeal Cbf5 and controls transitions between the closed and open states of the Cbf5 thumb loop (Duan et al. 2009; Wang et al. 2015). Although it seems that archaeal Gar1 makes no direct RNA contacts, the $\mathrm{N}$ - and C-terminal parts of eukaryotic Gar1 contains glycine-arginine-rich RGG domains (Girard et al. 1992). RGG domains are frequent in RNAbinding proteins and they play accessory roles in RNA binding (Thandapani et al. 2013). The RGG domains of yeast Garl have been found to facilitate pseudouridylation of the bound substrate RNA and to inhibit its release, raising the possibility that eukaryotic Garl may directly interact with target RNAs though its RGG domains (Trucks et al. 2021).

It seems that dual pseudouridylation guide loops are not confined to vertebrate H/ACA RNAs. Genetic depletion of yeast snR82 abolished pseudouridylation of the $27 \mathrm{~S}$ rRNA at U2350 and also at the nearby U2348 residue (Schattner et al. 2004). While synthesis of $\Psi 2350$ was predicted to be guided by the 3 '-terminal pseudouridylation loop of snR82, snR82-dependent synthesis of $\Psi 2348$ could not be reasoned, as U2348 was imbedded in the downstream antisense target stem (DATS) of snR 82 directing

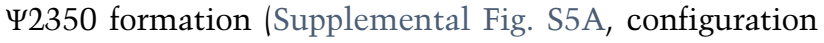
A). However, the $3^{\prime}$-terminal pseudouridylation loop of snR82 can form an alternative interaction with the $27 \mathrm{~S}$ rRNA to position U2348 for pseudouridylation (configuration $\mathrm{B})$. More recently, the $3^{\prime}$ hairpin of the archaeal
Haloferax volcanii sR-h45 H/ACA RNA has been shown to govern pseudouridylation of the $23 \mathrm{~S}$ rRNA at both $\Psi 1940$ and $\Psi 1942$ (Majumder et al. 2020). The investigators proposed that the dual guide activity of the $3^{\prime}$ hairpin of sR-h45 was due to its unusual interaction with 23S sequences, placing four unpaired ribosomal nucleotides, 1940-UAUG-1943, including the U1940 and U1942 substrate Us, into the pseudouridylation pocket of the $3^{\prime}$-terminal pseudouridylation loop (Supplemental Fig. S5B, configuration A). In fact, pseudouridylation pockets can accommodate more than two unpaired nucleotides at the base of the distal stem (De Zoysa et al. 2018). The investigators suggested that in this context, Cbf5 bound to the ACA box of sR-h45 was able to convert both the canonically positioned U1940 and the downstream unpaired U1942 into $\Psi$. Instead, we propose that sR-h45 forms an alternative interaction with 23S rRNA in order to specifically select U1942 for pseudouridylation (configuration B). Importantly, the results of the mutational analysis presented by the investigators are consistent with the existence and functional significance of configuration B supporting $\Psi 1942$ synthesis. For instance, the presence of U1940 in the 23S rRNA was required for the synthesis of $\Psi 1942$, supporting the importance of U1940 in formation of DATS in configuration B. In contrast, replacement of the U1942 residue failed to affect U1940 pseudouridylation, as formation of functional configuration A is independent from U1942. Based on these observations, we propose that the $3^{\prime}$ hairpins of yeast snR 82 and Haloferax sR-h45 RNAs carry dual pseudouridylation guide loops directing synthesis of two consecutive $\Psi$ s in the yeast 25S and archaeal 23S rRNAs through formation of alternative base-pairing interactions. Thus, H/ACA guide RNA acrobatics seems to be an evolutionarily widespread strategy for synthesis of two consecutive $\Psi$ s in vertebrate, yeast, and archaeal spliceosomal and ribosomal RNAs.

How many human H/ACA RNAs contain dual pseudouridylation guide loops? Apparently, synthesis of isolated $\Psi$ s dispersed in ribosomal and spliceosomal sequences is directed by specific pseudouridylation guide loops. Although it is unlikely that our search has overlooked many H/ACA RNAs with dual pseudouridylation guide loops (Fig. 5), it is possible that some pseudouridylation loops assigned for synthesis of $\Psi$ s in heavily pseudouridylated sequence environments possess dual guide capacity, as efficient synthesis of some $\Psi$ s might be accomplished by cooperative actions of single and dual pseudouridylation guide loops. Nevertheless, demonstration that four H/ACA RNAs carry dual pseudouridylation loops already shows that synthesis of at least $6 \%$ of the human ribosomal and spliceosomal $\Psi$ s is directed by dual guide loops, indicating that formation of closely located $\Psi$ s by dual pseudouridylation loops is a relatively frequent strategy of H/ACA RNPs.

In summary, guide RNA acrobatics supporting accurate and efficient conversion of nearby substrate Us (UU or UNU) into $\Psi$ is a novel, unexpected, relatively frequent, and, most probably, evolutionarily widespread strategy of box H/ACA pseudouridylation guide RNPs. 
Materials and methods

General procedures

Unless stated otherwise, all DNA and oligonucleotide manipulations were performed according to standard laboratory procedures. Oligodeoxynucleotides were purchased from Eurofins MWG Operon. Human HAP1 cells were grown in Iscove's modified Dulbecco's medium (IMDM) supplemented with 10\% (v/v) fetal bovine serum, $1 \mathrm{mM}$ sodium pyruvate (Gibco), and $100 \mu \mathrm{g} /$ $\mathrm{mL}$ penicillin and streptomycin (Gibco). Cells were transfected by electroporation with Gene Pulser (Bio-Rad) using exponential decay at settings $270 \mathrm{~V}$ and $950 \mu \mathrm{F}$.

\section{Expression plasmids}

Human HAP1 genomic fragments encompassing the SNORA53, SNORA57, SCARNA1, and SCARNA57 intronic H/ACA RNA genes were PCR-amplified by using sequence-specific primers encompassing the ClaI and XhoI restriction sites (Supplemental Table S1A). The resulting DNA fragments were inserted into the ClaI and XhoI sites of the pBST vector and the pCMV/globin expression vector (Darzacq et al. 2002). The H/ACA RNA gene together with its host globin gene was PCR-amplified and inserted into the EcoRI and NotI sites of pIRESpuro2 expression plasmid (Addgene). Mutant guide RNA genes were generated by the megaprimer PCR amplification approach using appropriately designed mutagenic oligonucleotide primers and wild-type sno/ scaRNA gene-containing recombinant pBST plasmids as templates. For nucleolar expression of mouse ribosomal minigene transcripts, appropriate oligonucleotides encoding the wild-type and mutant fragments of the human 28S rRNA from G3729 to G3759 were annealed and inserted into the XbaI and XhoI sites of the $\mathrm{pW}(\mathrm{Xb} / \mathrm{Xh})$ expression plasmid (Hadjiolova et al. 1994). The resulting $\mathrm{pW}-28 \mathrm{SWT}$ and $\mathrm{pW}-28 \mathrm{~S}-\mathrm{U} 3752 \mathrm{del}$ plasmids were transfected into 3T3 mouse cells with JetPrime transfection reagent (Polyplus Transfection SA). Two days after transfection, cellular RNA was extracted. The identities of all expression constructs were verified by sequence analysis.

\section{Excision of HAP1 intronic H/ACA RNA genes by CRISPR/Cas9}

To generate synthetic DNA encoding appropriately designed sgRNAs, appropriately designed oligodeoxynucleotides (Supplemental Table S1B) were annealed and inserted into the BbsI site of pSpCas9(BB)-2A-Puro (PX459) V2.0 by overnight incubation with T4 DNA ligase (NEB) at room temperature. The resulting pX459/sgRNA expression vectors were transfected into HAP1 cells. Twenty-four hours after transfection, cells were transferred into puromycin-containing $(1.5 \mu \mathrm{g} / \mathrm{mL})$ selective medium and grown for $48 \mathrm{~h}$ before establishment of monoclonal stable cell lines by limited dilution under nonselective conditions.

\section{Genomic PCR and RT-PCR}

Cellular DNA was purified with GenElute mammalian genomic DNA miniprep kit (Sigma), and the targeted genomic fragments were PCR-amplified with appropriately designed oligonucleotide primers (Supplemental Table S1C) and Phusion DNA polymerase (Thermo Scientific). cDNAs were generated with AMV reverse transcriptase (Promega) using total cellular RNAs as templates and sequence-specific oligonucleotide primers. The PCR-amplified genomic and cDNA fragments were analyzed by electrophoresis on $1 \%$ agarose gels.
RNA purification and primer extension mapping of pseudouridylation

The guanidine thiocyanate-phenol-chloroform purification procedure was used for RNA extraction from human HAP1 and HAP1-derived cells as described before (Kiss and Jády 2004). Cellular RNAs were reacted with $\mathrm{N}$-cyclohexyl-N'-(2-morpholinoethyl/carbodiimide metho-p-toluenesulfonate (CMCT) and subjected to primer extension analyses (Bakin and Ofengand 1998; Kiss and Jády 2004). The oligonucleotide primers used for pseudouridylation mapping of $18 \mathrm{~S}$ and $28 \mathrm{~S}$ rRNAs, U2 snRNA, and mouse ribosomal minigene transcripts are listed in Supplemental Table S1D.

RNase A/T1 protection analysis

RNase A and T1 protection analysis was performed according to Goodall et al. 1990. For mapping of endogenous and ectopically expressed wild-type and mutant SNORA53, SNORA57, SCARNA1, SCARNA8, and SCARNA14 RNAs, complementary RNA probes were synthesized in vitro by T3 RNA polymerase (Promega), using $30 \mu \mathrm{Ci}[\alpha-32 \mathrm{P}] \mathrm{CTP}$ and ClaI-linearized pBST-SNORA53, pBST-SNORA57, pBST-SCARNA1, pBSTSCARNA8, pBST-SCARNA14, and pBST-U85 plasmids as templates. Before hybridization with test RNAs, each probe was purified on a $6 \%$ denaturing gel. The protected fragments of the probe RNAs were analyzed on $6 \%$ sequencing gels.

\section{Competing interest statement}

The authors declare no competing interests.

\section{Acknowledgments}

Our research was supported by grants from l'Agence National de la Recherche (ANR-18-CE12-0008-01) and Institut National de la Santé et de la Recherche Médicale Plan Cancer to T.K.

Author contributions: B.E.J., A.K., and D.M. carried out experiments. B.E.J. and T.K. conceived and designed experiments and analyzed results. T.K. wrote the manuscript.

\section{References}

Adachi H, De Zoysa MD, Yu YT. 2019. Post-transcriptional pseudouridylation in mRNA as well as in some major types of noncoding RNAs. Biochim Biophys Acta Gene Regul Mech 1862: 230-239. doi:10.1016/j.bbagrm.2018.11.002

Arnez JG, Steitz TA. 1994. Crystal structure of unmodified tRNAGln complexed with glutaminyl-tRNA synthetase and ATP suggests a possible role for pseudo-uridines in stabilization of RNA structure. Biochemistry 33: 7560-7567. doi:10 $.1021 / \mathrm{bi00190a008}$

Baker DL, Youssef OA, Chastkofsky MI, Dy DA, Terns RM, Terns MP. 2005. RNA-guided RNA modification: functional organization of the archaeal H/ACA RNP. Genes Dev 19: 1238-1248. doi:10.1101/gad.1309605

Bakin AV, Ofengand J. 1998. Mapping of pseudouridine residues in RNA to nucleotide resolution. Methods Mol Biol 77: 297309.

Balakin AG, Smith L, Fournier MJ. 1996. The RNA world of the nucleolus: two major families of small RNAs defined by different box elements with related functions. Cell 86: 823-834. doi:10.1016/S0092-8674|00|80156-7 
Boccaletto P, Machnicka MA, Purta E, Piątkowski P, Bagiński B, Wirecki TK, de Crécy-Lagard V, Ross R, Limbach PA, Kotter A, et al. 2018. MODOMICS: a database of RNA modification pathways. 2017 update. Nucleic Acids Res 46: D303-D307. doi:10.1093/nar/gkx1030

Bohnsack MT, Sloan KE. 2018. Modifications in small nuclear RNAs and their roles in spliceosome assembly and function. Biol Chem 399: 1265-1276. doi:10.1515/hsz-2018-0205

Carlile TM, Rojas-Duran MF, Zinshteyn B, Shin H, Bartoli KM, Gilbert WV. 2014. Pseudouridine profiling reveals regulated mRNA pseudouridylation in yeast and human cells. Nature 515: 143-146. doi:10.1038/nature 13802

Czekay DP, Kothe U. 2021. H/ACA small ribonucleoproteins: structural and functional comparison between archaea and eukaryotes. Front Microbiol 12: 654370. doi:10.3389/fmicb .2021 .654370

Darzacq X, Jady BE, Verheggen C, Kiss AM, Bertrand E, Kiss T. 2002. Cajal body-specific small nuclear RNAs: a novel class of 2'-O-methylation and pseudouridylation guide RNAs. EMBO I 21: 2746-2756. doi:10.1093/emboj/21.11.2746

Davis DR. 1995. Stabilization of RNA stacking by pseudouridine. Nucleic Acids Res 23: 5020-5026. doi:10.1093/nar/23.24.5020

Deryusheva S, Gall JG. 2013. Novel small cajal-body-specific RNAs identified in Drosophila: probing guide RNA function. RNA 19: 1802-1814. doi:10.1261/rna.042028.113

Deryusheva S, Gall JG. 2017. Dual nature of pseudouridylation in U2 snRNA: Pus1p-dependent and Pus1p-independent activities in yeasts and higher eukaryotes. RNA 23: 1060-1067. doi:10.1261/rna.061226.117

De Zoysa MD, Yu YT. 2017. Posttranscriptional RNA pseudouridylation. Enzymes 41: 151-167. doi:10.1016/bs.enz.2017.02 .001

De Zoysa MD, Wu G, Katz R, Yu YT. 2018. Guide-substrate basepairing requirement for box H/ACA RNA-guided RNA pseudouridylation. RNA 24: 1106-1117. doi:10.1261/rna.066837 .118

Duan J, Li L, Lu J, Wang W, Ye K. 2009. Structural mechanism of substrate RNA recruitment in H/ACA RNA-guided pseudouridine synthase. Mol Cell 34: 427-439. doi:10.1016/j.molcel .2009 .05 .005

Egan ED, Collins K. 2010. Specificity and stoichiometry of subunit interactions in the human telomerase holoenzyme assembled in vivo. Mol Cell Biol 30: 2775-2786. doi:10.1128/ MCB.00151-10

Fernández IS, Ng CL, Kelley AC, Wu G, Yu YT, Ramakrishnan V. 2013. Unusual base pairing during the decoding of a stop codon by the ribosome. Nature 500: 107-110. doi:10.1038/ nature 12302

Ganot P, Bortolin ML, Kiss T. 1997a. Site-specific pseudouridine formation in preribosomal RNA is guided by small nucleolar RNAs. Cell 89: 799-809. doi:10.1016/S0092-8674|00|80263-9

Ganot P, Caizergues-Ferrer M, Kiss T. 1997b. The family of box ACA small nucleolar RNAs is defined by an evolutionarily conserved secondary structure and ubiquitous sequence elements essential for RNA accumulation. Genes Dev 11: 941956. doi:10.1101/gad.11.7.941

Ge J, Yu YT. 2013. RNA pseudouridylation: new insights into an old modification. Trends Biochem Sci 38: 210-218. doi:10 .1016/j.tibs.2013.01.002

Girard JP, Lehtonen H, Caizergues-Ferrer M, Amalric F, Tollervey D, Lapeyre B. 1992. GAR1 is an essential small nucleolar RNP protein required for pre-rRNA processing in yeast. Embo $j$ 11: 673-682. doi:10.1002/j.1460-2075.1992.tb05099.x
Goodall GJ, Wiebauer K, Filipowicz W. 1990. Analysis of premRNA processing in transfected plant protoplasts. Methods Enzymol 181: 148-161. doi:10.1016/0076-6879(90)81117-d

Hadjiolova KV, Normann A, Cavaille J, Soupene E, Mazan S, Hadjiolov AA, Bachellerie JP. 1994. Processing of truncated mouse or human rRNA transcribed from ribosomal minigenes transfected into mouse cells. Mol Cell Biol 14: 4044-4056.

Hoernes TP, Clementi N, Faserl K, Glasner H, Breuker K, Lindner H, Hüttenhofer A, Erlacher MD. 2016. Nucleotide modifications within bacterial messenger RNAs regulate their translation and are able to rewire the genetic code. Nucleic Acids Res 44: 852-862. doi:10.1093/nar/gkv1182

Jorjani H, Kehr S, Jedlinski DJ, Gumienny R, Hertel J, Stadler PF, Zavolan M, Gruber AR. 2016. An updated human snoRNAome. Nucleic Acids Res 44: 5068-5082. doi:10 .1093/nar/gkw386

Karijolich J, Yu YT. 2011. Converting nonsense codons into sense codons by targeted pseudouridylation. Nature 474: 395-398. doi:10.1038/nature10165

Karikó K, Muramatsu H, Welsh FA, Ludwig J, Kato H, Akira S, Weissman D. 2008. Incorporation of pseudouridine into mRNA yields superior nonimmunogenic vector with increased translational capacity and biological stability. Mol Ther 16: 1833-1840. doi:10.1038/mt.2008.200

Kiss T, Jády BE. 2004. Functional characterization of 2'-O-methylation and pseudouridylation guide RNAs. Methods Mol Biol 265: 393-408.

Kiss T, Bortolin ML, Filipowicz W. 1996. Characterization of the intron-encoded U19 RNA, a new mammalian small nucleolar RNA that is not associated with fibrillarin. Mol Cell Biol 16: 1391-1400. doi:10.1128/MCB.16.4.1391

Kiss AM, Jády BE, Bertrand E, Kiss T. 2004. Human box H/ACA pseudouridylation guide RNA machinery. Mol Cell Biol 24: 5797-5807. doi:10.1128/MCB.24.13.5797-5807.2004

Kiss T, Fayet-Lebaron E, Jády BE. 2010. Box H/ACA small ribonucleoproteins. Mol Cell 37: 597-606. doi:10.1016/j.molcel.2010 .01 .032

Lestrade L, Weber MJ. 2006. snoRNA-LBME-db, a comprehensive database of human H/ACA and C/D box snoRNAs. Nucleic Acids Res 34: D158-D162. doi:10.1093/nar/gkj002

Li L, Ye K. 2006. Crystal structure of an H/ACA box ribonucleoprotein particle. Nature 443: 302-307. doi:10.1038/ nature 05151

Li S, Duan J, Li D, Yang B, Dong M, Ye K. 2011. Reconstitution and structural analysis of the yeast box H/ACA RNA-guided pseudouridine synthase. Genes Dev 25: 2409-2421. doi:10 $.1101 /$ gad.175299.111

Li X, Zhu P, Ma S, Song J, Bai J, Sun F, Yi C. 2015. Chemical pulldown reveals dynamic pseudouridylation of the mammalian transcriptome. Nat Chem Biol 11: 592-597. doi:10.1038/ nchembio. 1836

Liang B, Xue S, Terns RM, Terns MP, Li H. 2007. Substrate RNA positioning in the archaeal H/ACA ribonucleoprotein complex. Nat Struct Mol Biol 14: 1189-1195. doi:10.1038/ nsmb1336

Liang B, Zhou J, Kahen E, Terns RM, Terns MP, Li H. 2009. Structure of a functional ribonucleoprotein pseudouridine synthase bound to a substrate RNA. Nat Struct Mol Biol 16: 740-746. doi: $10.1038 / \mathrm{nsmb} .1624$

Lui L, Lowe T. 2013. Small nucleolar RNAs and RNA-guided post-transcriptional modification. Essays Biochem 54: 5377. doi:10.1042/bse0540053

Majumder M, Mukhopadhyay S, Kharel P, Gupta R. 2020. The presence of the ACA box in archaeal H/ACA guide RNAs 
promotes atypical pseudouridylation. RNA 26: 396-418. doi:10.1261/rna.073734.119

Newby MI, Greenbaum NL. 2002a. Investigation of overhauser effects between pseudouridine and water protons in RNA helices. Proc Natl Acad Sci 99: 12697-12702. doi:10.1073/pnas .202477199

Newby MI, Greenbaum NL. 2002b. Sculpting of the spliceosomal branch site recognition motif by a conserved pseudouridine. Nat Struct Biol 9: 958-965. doi:10.1038/nsb873

Ni J, Tien AL, Fournier MJ. 1997. Small nucleolar RNAs direct site-specific synthesis of pseudouridine in ribosomal RNA. Cell 89: 565-573. doi:10.1016/S0092-8674(00)80238-X

Reddy R, Busch H. 1988. Small nuclear RNAs: RNA sequences, structure and modifications. In Structure and function of major and minor small nuclear ribonucleoprotein (ed. Birnstiel ML), pp. 1-37. Springer-Verlag Press, Berlin, Germany.

Schattner P, Decatur WA, Davis CA, Ares M Jr., Fournier MJ, Lowe TM. 2004. Genome-wide searching for pseudouridylation guide snoRNAs: analysis of the Saccharomyces cerevisiae genome. Nucleic Acids Res 32: 4281-4296. doi:10.1093/ nar/gkh768

Schattner P, Barberan-Soler S, Lowe TM. 2006. A computational screen for mammalian pseudouridylation guide H/ACA RNAs. RNA 12: 15-25. doi:10.1261/rna.2210406

Schwartz S, Bernstein DA, Mumbach MR, Jovanovic M, Herbst RH, León-Ricardo BX, Engreitz JM, Guttman M, Satija R, Lander ES, et al. 2014. Transcriptome-wide mapping reveals widespread dynamic-regulated pseudouridylation of ncRNA and mRNA. Cell 159: 148-162. doi:10.1016/j.cell .2014.08.028

Sloan KE, Warda AS, Sharma S, Entian KD, Lafontaine DLJ, Bohnsack MT. 2017. Tuning the ribosome: the influence of rRNA modification on eukaryotic ribosome biogenesis and function. RNA Biol 14: 1138-1152. doi:10.1080/15476286 .2016 .1259781
Spenkuch F, Motorin Y, Helm M. 2014. Pseudouridine: still mysterious, but never a fake (uridine)!. RNA Biol 11: 1540-1554. doi:10.4161/15476286.2014.992278

Taoka M, Nobe Y, Yamaki Y, Sato K, Ishikawa H, Izumikawa K, Yamauchi Y, Hirota K, Nakayama H, Takahashi N, et al. 2018. Landscape of the complete RNA chemical modifications in the human $80 \mathrm{~S}$ ribosome. Nucleic Acids Res 46: 9289-9298. doi:10.1093/nar/gky811

Thandapani P, O'Connor TR, Bailey TL, Richard S. 2013. Defining the RGG/RG motif. Mol Cell 50: 613-623. doi:10.1016/j .molcel.2013.05.021

Trucks S, Hanspach G, Hengesbach M. 2021. Eukaryote specific RNA and protein features facilitate assembly and catalysis of H/ACA snoRNPs. Nucleic Acids Res 49: 4629-4642. doi:10.1093/nar/gkab177

Vitali P, Kiss T. 2019. Cooperative 2'-O-methylation of the wobble cytidine of human elongator tRNA ${ }^{\text {Met }}(\mathrm{CAT})$ by a nucleolar and a Cajal body-specific box C/D RNP. Genes Dev 33: 741746. doi:10.1101/gad.326363.119

Wang C, Meier UT. 2004. Architecture and assembly of mammalian H/ACA small nucleolar and telomerase ribonucleoproteins. EMBO / 23: 1857-1867. doi:10.1038/sj.emboj.7600181

Wang P, Yang L, Gao YQ, Zhao XS. 2015. Accurate placement of substrate RNA by Gar1 in H/ACA RNA-guided pseudouridylation. Nucleic Acids Res 43: 7207-7216. doi:10.1093/nar/ gkv757

Watkins NJ, Bohnsack MT. 2012. The box C/D and H/ACA snoRNPs: key players in the modification, processing and the dynamic folding of ribosomal RNA. Wiley Interdiscip Rev RNA 3: 397-414. doi:10.1002/wrna.117

Yu YT, Meier UT. 2014. RNA-guided isomerization of uridine to pseudouridine-pseudouridylation. RNA Biol 11: 1483-1494. doi:10.4161/15476286.2014.972855

Zuker M. 2003. Mfold web server for nucleic acid folding and hybridization prediction. Nucleic Acids Res 31: 3406-3415. doi:10.1093/nar/gkg595 


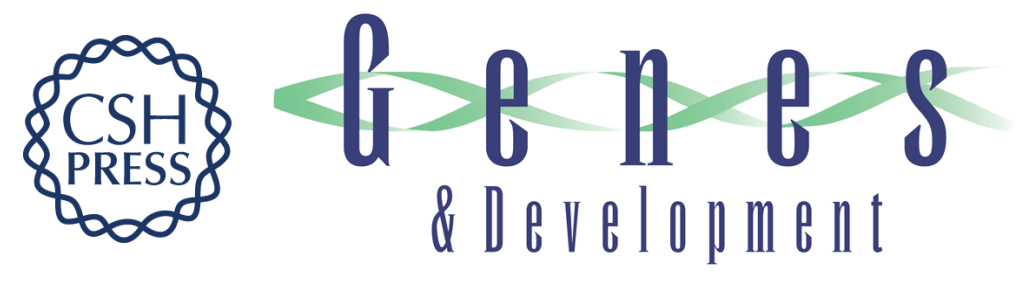

\section{Guide RNA acrobatics: positioning consecutive uridines for pseudouridylation by H/ACA pseudouridylation loops with dual guide capacity}

Beáta E. Jády, Amandine Ketele, Dylan Moulis, et al.

Genes Dev. 2022, 36: originally published online December 16, 2021

Access the most recent version at doi:10.1101/gad.349072.121

\section{Supplemental http://genesdev.cshlp.org/content/suppl/2021/12/15/gad.349072.121.DC1 \\ Material}

Related Content Guide RNA acrobatics: the one-for-two shuffle

U. Thomas Meier

Genes Dev. January , 2022 36: 1-3

References This article cites 57 articles, 17 of which can be accessed free at:

http://genesdev.cshlp.org/content/36/1-2/70.full.html\#ref-list-1

Articles cited in:

http://genesdev.cshlp.org/content/36/1-2/70.full.htmI\#related-urls

Creative This article, published in Genes \& Development, is available under a Creative Commons

Commons

License

License (Attribution-NonCommercial 4.0 International), as described at

http://creativecommons.org/licenses/by-nc/4.0/.

Email Alerting

Receive free email alerts when new articles cite this article - sign up in the box at the top

Service right corner of the article or click here.

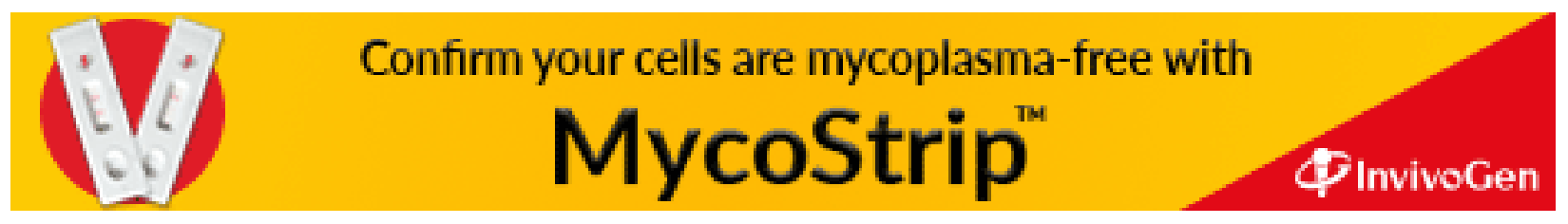

